\title{
On moments of a polytope
}

\author{
Nick Gravin ${ }^{1}$ - Dmitrii V. Pasechnik ${ }^{2} \cdot$ Boris Shapiro $^{3}$ (D) \\ Michael Shapiro ${ }^{4}$
}

Received: 12 December 2017 / Accepted: 2 April 2018 / Published online: 12 April 2018 (C) The Author(s) 2018

\begin{abstract}
We show that the multivariate generating function of appropriately normalized moments of a measure with homogeneous polynomial density supported on a compact polytope $\mathcal{P} \subset \mathbb{R}^{d}$ is a rational function. Its denominator is the product of linear forms dual to the vertices of $\mathcal{P}$ raised to the power equal to the degree of the density function. Using this, we solve the inverse moment problem for the set of, not necessarily convex, polytopes having a given set $S$ of vertices. Under a weak nondegeneracy assumption we also show that the uniform measure supported on any such polytope is a linear combination of uniform measures supported on simplices with vertices in $S$.
\end{abstract}

To the memory of Mikael Passare.

$凶$ Boris Shapiro

shapiro@math.su.se

Nick Gravin

nikolai@mail.shufe.edu.cn

Dmitrii V. Pasechnik

dimpase@cs.ox.ac.uk

Michael Shapiro

mshapiro@math.msu.edu

1 Shanghai University of Finance and Economics, 100 Wudong Road, Yangpu District, Shanghai, China

2 Department of Computer Science, University of Oxford, Wolfson Building, Parks Road, Oxford, OX1 3QD, UK

3 Department of Mathematics, Stockholm University, 10691 Stockholm, Sweden

4 Department of Mathematics, Michigan State University, East Lansing, MI 48824-1027, USA 
Keywords Moments of polyhedra - Generating functions $\cdot$ Hyperplane arrangements Moment problems

Mathematics Subject Classification Primary 44A60; Secondary 31B20

\section{Introduction}

The initial motivation for the present paper came from proposed in [20] efficient algorithm recovering an arbitrary convex polytope from axial moments of a polynomial measure supported on it. This algorithm is based on the formulas for the axial moments of polytopes found over 20 years ago independently by Brion, Lawrence, Khovanskii, Pukhlikov, and Barvinok [7, 12,22,27], see [8,9] for accessible explanation. In [20] the authors made an essential, although implicit, use of a univariate rational generating function for appropriately normalized axial moments. Here a multivariate, and explicit, analog of the latter function is developed. It turns out it provides a very convenient encoding of non-convex polytopes, which is of independent interest. E.g. it leads to a natural definition of vertices of such non-convex polytopes, which have similar properties to vertices of convex polytopes. It also allows to find the exact solutions of a class of inverse moment problems on non-convex polytopes.

After the first version [19] of this text was released in 2012, it was pointed out to us by Prof. Michèle Vergne that Laplace transform techniques developed for studying hyperplane arrangements in [13] simplify and strengthen a number of our results. We discuss this in the Sect. 5.1, while leaving full details for another publication.

Notation 1 In what follows we shall always assume that $\mathbb{R}^{d}$ is endowed with a fixed coordinate system $\left(x_{1}, \ldots, x_{d}\right)$, orthonormal with respect to the standard scalar product $\langle\cdot, \cdot\rangle$. Let $\mu$ be a finite complex-valued Borel measure in $\mathbb{R}^{d}$. (For standard measure-theoretic notions we follow [29].) Given a multiindex $I=\left(i_{1}, \ldots, i_{d}\right)$, let $\mathbf{x}^{I}$ be the shorthand of the monomial $x_{1}^{i_{1}} \ldots x_{d}^{i_{d}}$ and $|I|$ the shorthand for $i_{1}+\cdots+i_{d}$. For any multiindex $I$, define the moment $m_{I}(\mu)$ of $\mu$ as

$$
m_{I}(\mu):=\int_{\mathbb{R}^{d}} x_{1}^{i_{1}} x_{2}^{i_{2}} \ldots x_{d}^{i_{d}} d \mu\left(x_{1}, x_{2}, \ldots, x_{d}\right)=\int_{\mathbb{R}^{d}} \mathbf{x}^{I} d \mu(\mathbf{x}) .
$$

Define the normalized moment generating function $F_{\mu}(\mathbf{u})=F_{\mu}\left(u_{1}, \ldots, u_{d}\right)$ of $\mu$ by

$$
F_{\mu}(\mathbf{u}):=\sum_{I:=\left(i_{1}, \ldots, i_{d}\right) \geq 0} \frac{(|I|+d) !}{i_{1} ! \cdots i_{d} !} m_{I}(\mu) \mathbf{u}^{I}, \quad \text { where } \mathbf{u}^{I}=u_{1}^{i_{1}} \ldots u_{d}^{i_{d}} .
$$

Note that $F_{\mu}(\mathbf{u})$ admits the integral representation

$$
F_{\mu}(\mathbf{u})=d ! \int_{\mathbb{R}^{d}} \frac{d \mu(\mathbf{x})}{(1-\langle\mathbf{x}, \mathbf{u}\rangle)^{d+1}},
$$

which is a special case of a Fantappiè transformation. For details on the latter, see e.g. [5, Chapter 3]. A proof of (1.3) will be given at the end of Sect. 2; see also Remark 10. 
Given any complex-valued finite measure $\mu$ and any degree $\delta$ homogeneous $d$ variate polynomial $\rho$, it is convenient to define the (re)normalized moment generating function $F_{\mu}^{\rho}$ (u) for the measure $\rho \mu$, where by definition, $\int_{\mathbb{R}^{d}} f d(\rho \mu)=\int_{\mathbb{R}^{d}} f \rho d \mu$, in such a way that it can be obtained from $F_{\mu}(u)$ by application of the differential operator $\rho\left(\frac{\partial}{\partial \mathbf{u}}\right)$. Namely, set

$$
F_{\mu}^{\rho}(\mathbf{u}):=\sum_{I:=\left(i_{1}, \ldots, i_{d}\right) \geq 0} \frac{(|I|+d+\delta) !}{i_{1} ! \cdots i_{d} !} m_{I}(\rho \mu) \mathbf{u}^{I} .
$$

Note that $F_{\mu}^{\rho}(\mathbf{u}) \neq F_{\rho \mu}(\mathbf{u})$ for non-constant $\rho$. However, they are also connected, by an explicit differential operator as follows.

Theorem 1 For any complex-valued finite measure $\mu$ and any homogeneous polynomial $\rho$ of degree $\delta$,

$$
\begin{aligned}
F_{\mu}^{\rho}(\mathbf{u}) & =\prod_{\ell=d}^{d+\delta-1}\left(\sum_{k} u_{k} \frac{\partial}{\partial u_{k}}+\ell\right) \circ F_{\rho \mu}(\mathbf{u}) \\
& =\rho\left(\frac{\partial}{\partial \mathbf{u}}\right) \circ F_{\mu}(\mathbf{u}) \\
& =(d+\delta) ! \int_{\mathbb{R}^{d}} \frac{\rho(\mathbf{x}) d \mu(\mathbf{x})}{(1-\langle\mathbf{x}, \mathbf{u}\rangle)^{d+\delta+1}} .
\end{aligned}
$$

Here and in what follows $\circ$ denotes the application of a differential operator to a function. The proof of the latter result is basically an exercise in manipulating formal power series, and we do not claim its novelty. For the sake of completeness, we include a proof in Sect. 2.

\section{Results on convex polytopes}

A finite set $S \subset \mathbb{R}^{d}$ is called spanning if it is not contained in any (affine) hyperplane in $\mathbb{R}^{d}$. (Obviously, card $(S) \geq d+1$.) As usual, by a (compact, convex) polytope $\mathcal{P} \subset \mathbb{R}^{d}$ we mean the convex hull of a finite spanning set in $\mathbb{R}^{d}$. The set of vertices of a convex polytope $\mathcal{P}$ is the inclusion-minimal finite set with convex hull $\mathcal{P}$. A $d$-simplex in $\mathbb{R}^{d}$ is the convex hull of a spanning $(d+1)$-tuple of points. By an open polytope (resp. simplex) we mean the set of interior points of a compact polytope (resp. simplex).

Given a convex polytope $\mathcal{P}$ let $\mathcal{V}=\left(\mathbf{v}_{1}, \ldots, \mathbf{v}_{N}\right)$ denote the set of its vertices. Assume that $\mathcal{P}$ is simple, i.e. each $\mathbf{v} \in \mathcal{V}$ has exactly $d$ incident edges $\mathbf{v v}_{e_{1}}$, $\ldots, \mathbf{v v}_{e_{d}}$. Set $w_{k}(\mathbf{v}):=\mathbf{v}_{e_{k}}-\mathbf{v}$, for $1 \leq k \leq d$. The non-negative real span $K_{\mathbf{v}}$ of $w_{1}(\mathbf{v}), \ldots, w_{d}(\mathbf{v})$ is called the tangent cone of $\mathcal{P}$ at $\mathbf{v}$. For each $K_{\mathbf{v}}$, define $\left|\operatorname{det} K_{\mathbf{v}}\right|=\left|\operatorname{det}\left(w_{1}(\mathbf{v}), \ldots, w_{d}(\mathbf{v})\right)\right|$ to be the volume of the parallelepiped formed by $w_{1}(\mathbf{v}), \ldots, w_{d}(\mathbf{v})$.

Given a bounded domain $\Omega \subset \mathbb{R}^{d}$, we call the measure

$$
\mu_{\Omega}=\chi_{\Omega} d x_{1} d x_{2} \ldots d x_{d}
$$


where $\chi_{\Omega}$ is the characteristic function of $\Omega$, the standard measure of $\Omega$.

For a simple convex polytope $\mathcal{P}$, we have the following explicit representation of $F_{\mu_{\mathcal{P}}}(\mathbf{u})$.

Theorem 2 For an arbitrary simple convex polytope $\mathcal{P}$,

$$
\begin{aligned}
F_{\mathcal{P}}(\mathbf{u}):=F_{\mu_{\mathcal{P}}}(\mathbf{u}) & =(-1)^{d} \sum_{\mathbf{v} \in \mathcal{V}} \frac{\langle\mathbf{v}, \mathbf{u}\rangle^{d}\left|\operatorname{det} K_{\mathbf{v}}\right|}{\prod_{j=1}^{d}\left\langle w_{j}(\mathbf{v}), \mathbf{u}\right\rangle} \cdot \frac{1}{1-\langle\mathbf{v}, \mathbf{u}\rangle} \\
& =(-1)^{d} \sum_{\mathbf{v} \in \mathcal{V}} \frac{\left|\operatorname{det} K_{\mathbf{v}}\right|}{\prod_{j=1}^{d}\left\langle w_{j}(\mathbf{v}), \mathbf{u}\right\rangle} \cdot \frac{1}{1-\langle\mathbf{v}, \mathbf{u}\rangle}
\end{aligned}
$$

Remark 1 Instead of the explicit choice of $w_{k}(\mathbf{v})$ for $\mathbf{v} \in \mathcal{V}$ made above, we can take any fixed set of non-zero vectors $w_{1}(\mathbf{v}), \ldots, w_{d}(\mathbf{v})$, spanning the tangent cone of $\mathbf{v}$ in $\mathcal{P}$. This does not affect the validity of (1.8) and (1.9).

Theorem 2 implies

Corollary 3 Let $\Delta=\operatorname{conv}(\mathcal{V}) \subset \mathbb{R}^{d}$ be an arbitrary $d$-simplex. Then

$$
F_{\Delta}(\mathbf{u})=\frac{d ! \operatorname{Vol}(\Delta)}{\prod_{\mathbf{v} \in \mathcal{V}}(1-\langle\mathbf{v}, \mathbf{u}\rangle)}
$$

Remark 2 As we discovered after we proved the above results, statements similar to Corollary 3 in the complex setting can be found in [5, Sect. 3.5] and in particular [5, Corollary 3.5.6].

A variation of (1.10) also appears in [6], in the context of designing an efficient procedure for integration of polynomials over simplices.

Notice that an arbitrary convex polytope $\mathcal{P}$ admits a triangulation which only uses the existing vertices of $\mathcal{P}$, see e.g. [9, Theorem 3.1]. Applying Corollary 3 and Theorem 1 to the sum of measures corresponding to such a triangulation we get the following.

Corollary 4 The normalized moment generating function $F_{\mathcal{P}}^{\rho}(\mathbf{u})$ of any convex polytope $\mathcal{P}$ with respect to any homogeneous polynomial density function $\rho$ of degree $\delta$ is a rational function with denominator dividing

$$
\prod_{\mathbf{v} \in \mathcal{V}}(1-\langle\mathbf{v}, \mathbf{u}\rangle)^{\delta}
$$

Example 1 Let $\Delta$ be a triangle in $\mathbb{R}^{2}$ with vertices $v_{1}=(1,1), v_{2}=(2,5)$ and $v_{3}=(3,2)$. Its normalized moment generating function equals

$$
F_{\Delta}\left(u_{1}, u_{2}\right)=\frac{7}{\left(1-u_{1}-u_{2}\right)\left(1-2 u_{1}-5 u_{2}\right)\left(1-3 u_{1}-2 u_{2}\right)} .
$$


Its Taylor expansion about the origin up to the terms of degree 7 is given by

$$
\begin{aligned}
7 & +42 u_{1}+56 u_{2}+175 u_{1}^{2}+455 u_{1} u_{2}+329 u_{2}^{2}+630 u_{1}^{3}+2387 u_{1}^{2} u_{2} \\
& +3367 u_{1} u_{2}^{2}+1750 u_{2}^{3}+2107 u_{1}^{4}+10318 u_{1}^{3} u_{2} \\
& +21217 u_{1}^{2} u_{2}^{2}+21546 u_{1} u_{2}^{3}+8967 u_{2}^{4}+6762 u_{1}^{5}+40082 u_{1}^{4} u_{2} \\
& +106526 u_{1}^{3} u_{2}^{2}+157976 u_{1}^{2} u_{2}^{3}+128772 u_{1} u_{2}^{4} \\
& +45276 u_{2}^{5}+21175 u_{1}^{6}+145845 u_{1}^{5} u_{2}+468895 u_{1}^{4} u_{2}^{2} \\
& +900123 u_{1}^{3} u_{2}^{3}+10744451 u_{1}^{2} u_{2}^{4}+741993 u_{1} u_{2}^{5}+227269 u_{2}^{6},
\end{aligned}
$$

which implies that

$$
\begin{aligned}
& m_{00}=\frac{7}{2}, m_{10}=7, m_{01}=\frac{28}{3}, m_{20}=\frac{175}{12}, m_{11}=\frac{455}{24}, \\
& m_{02}=\frac{329}{12}, m_{30}=\frac{63}{2}, m_{21}=\frac{2387}{60}, \\
& m_{12}=\frac{3591}{20}, m_{03}=\frac{175}{2}, m_{40}=\frac{2107}{30}, m_{31}=\frac{5159}{60}, \\
& m_{22}=\frac{21217}{180}, m_{13}=\frac{3591}{20}, m_{04}=\frac{2989}{10}, \\
& m_{50}=161, m_{41}=\frac{2863}{15}, m_{32}=\frac{7609}{30}, m_{23}=\frac{5642}{15}, \\
& m_{14}=\frac{3066}{5}, m_{05}=1078, m_{60}=\frac{3025}{8}, \\
& m_{51}=\frac{6945}{16}, m_{42}=\frac{13397}{24}, m_{33}=\frac{128589}{160}, \\
& m_{24}=\frac{153493}{120}, m_{15}=\frac{35333}{16}, m_{06}=\frac{32467}{8} .
\end{aligned}
$$

\section{Results on non-convex polytopes}

Our second group of results addresses the problem of distinguishing different polytopes with the same underlying set of vertices from information on their moments. The problem of restoring the vertices of a polygon or a polytope with a constant mass density from information on its moments was addressed earlier in e.g. [14, 18,20,21, $24,31]$. However, the latter do not provide the recovery of the vertices in the generality required in the present paper. Below we concentrate on the case of constant density and known vertices, and plan to return to the general inverse problem for polytopes with unknown polynomial density and unknown location of their vertices in the future.

First we need to define what we mean by a polytope. It turned out that there is no general consensus about this notion. Instead there exist several competing definitions having their own advantages in different situations. We shall study the following class of polytopal objects. 
Definition 1 A subset $\mathcal{P} \subset \mathbb{R}^{d}$ coinciding with a finite union of arbitrary convex $d$-dimensional polytopes is called a generalized polytope.

Definition 2 The number of components of a generalized polytope $\mathcal{P}$ is the number of connected components of the set $\mathcal{P}^{o} \subset \mathcal{P}$ of interior points of $\mathcal{P}$. The closure of each connected component of $\mathcal{P}^{o}$ is called a component of $\mathcal{P}$. A generalized polytope with one component is called indecomposable.

Remark 3 We say that a simplicial complex in $\mathbb{R}^{d}$ is pure if all its maximal simplices have dimension $d$. Clearly any generalized polytope in $\mathbb{R}^{d}$ can be represented as the topological space of an appropriate pure simplicial complex.

Remark 4 Often one considers a more restricted class of objects, namely polytopes. A polytope $\mathcal{P} \subset \mathbb{R}^{d}$ is a generalized polytope homeomorphic to a $d$-dimensional manifold with boundary.

We need to introduce the notion of a vertex of a generalized polytope.

Definition 3 Given a generalized polytope $\mathcal{P} \subset \mathbb{R}^{d}$, we call a finite collection of open disjoint $d$-dimensional simplices in $\mathbb{R}^{d}$ a dissection of $\mathcal{P}$ if the closure of their union coincides with $\mathcal{P}$.

A wealth of material on dissections of polytopes can be found in [25], see also [16].

Definition 4 Given a generalized polytope $\mathcal{P} \subset \mathbb{R}^{d}$, we call a point $\mathbf{v}$ a vertex of $\mathcal{P}$, if $\mathbf{v}$ is a vertex of (the closure of) some open simplex in every dissection of $\mathcal{P}$.

Definition 5 Given a point $p \in \mathcal{P}$ of a generalized polytope $\mathcal{P}$, we denote by the tangent cone $T_{p}(\mathcal{P})$ of $\mathcal{P}$ at $p$ the set obtained as follows. For a sufficiently small $\epsilon>0$, set $\mathcal{P}_{p}(\epsilon)=\mathcal{P} \cap B_{p}(\epsilon)$ where $B_{p}(\epsilon)$ is the $\epsilon$-ball centered at $p$. Define $T_{p}(\mathcal{P})$ as the set obtained by taking a ray through $p$ and every point of $\mathcal{P}_{p}(\epsilon)$. In other words, $T_{p}(\mathcal{P})$ is the cone with the apex at $p$ and the base $B_{p}(\epsilon)$. (Obviously, $T_{p}(\mathcal{P})$ is independent of $\epsilon$ for a sufficiently small $\epsilon>0$, and it need not be convex.)

Lemma 5 A point $\mathbf{v}$ is a vertex of $\mathcal{P}$ if and only if $T_{\mathbf{v}}(\mathcal{P})$ does not admit a decomposition in the disjoint union of convex polyhedral subcones, such that each subcone in the decomposition has a translation-invariant direction (i.e. is not pointed). In particular, if the tangent cone to $\mathcal{P}$ at $\mathbf{v}$ has a connected component with no translation-invariant direction, then $\mathbf{v}$ is a vertex.

We denote by conv $(S)$ the convex hull of an arbitrary set $S \subset \mathbb{R}^{d}$. The above lemma implies that any vertex of $\operatorname{conv}(\mathcal{P})$ is a vertex of $\mathcal{P}$.

The following result extends Corollary 4 to the case of generalized polytopes.

Proposition 6 For any generalized polytope $\mathcal{P}$ with the set of vertices $\mathcal{V}(\mathcal{P})$, the denominator of its normalized moment generating function $F_{\mathcal{P}}^{\rho}(\mathbf{u})$ with respect to a homogeneous polynomial density function $\rho$ of degree $\delta$ divides

$$
\Phi_{\mathcal{P}}(\mathbf{u}):=\prod_{\mathbf{v} \in \mathcal{V}(\mathcal{P})}(1-\langle\mathbf{v}, \mathbf{u}\rangle)^{\delta} .
$$



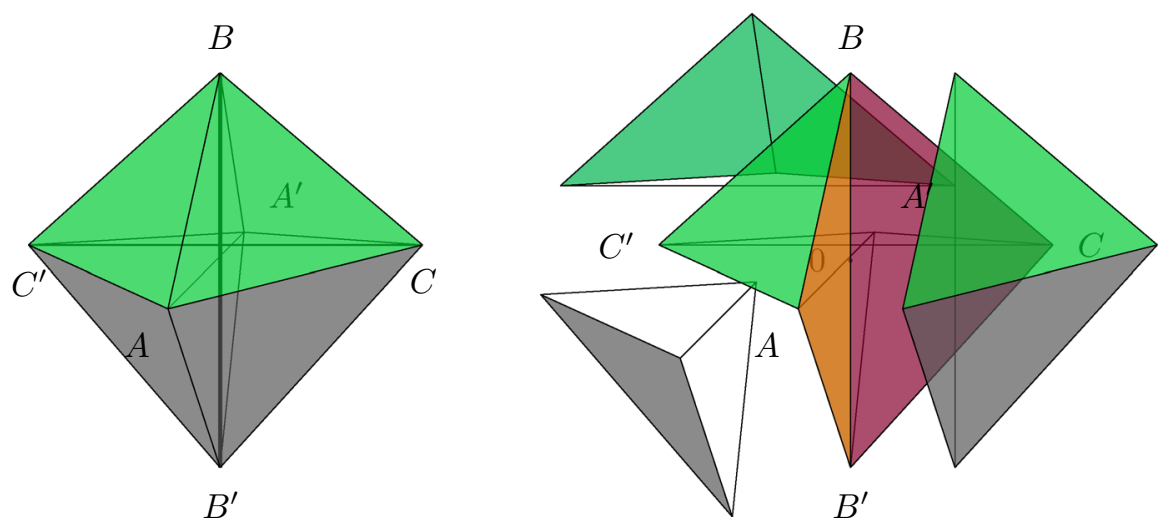

Fig. 1 Schönhardt polyhedron obtained from an octahedron (on the left) by removing tetrahedra $\left[A B B^{\prime} C\right]$, $\left[A A^{\prime} B^{\prime} C^{\prime}\right]$, and $\left[A^{\prime} B C C^{\prime}\right]$

Remark 5 There exist generalized polytopes which do not admit dissections with only existing vertices. The simplest example of this kind is the Schönhardt polyhedron, see Fig. 1 and [30]. Absence of a dissection $\mathcal{T}$ which uses only its 6 vertices can be established by observing that none of the edges $A C, A^{\prime} B$, and $B^{\prime} C^{\prime}$ can appear in a simplex of $\mathcal{T}$, yet any simplex on these 6 vertices must contain one of them. Therefore, Proposition 6 is not an immediate consequence of Corollary 3.

Remark 6 For "generic" generalized polytopes $\mathcal{P}$, the denominator $\Omega(\mathbf{u})$ of $F_{\mathcal{P}}(\mathbf{u})$ equals $\Phi_{\mathcal{P}}(\mathbf{u})$, but for certain special polytopes the denominator $\Omega(\mathbf{u})$ may be its proper divisor, as can be seen from the following example. Let $A=\left\{0, a_{1}, a_{2}, a_{3}\right\} \subset$ $\mathbb{R}^{3}$ be a spanning set, and $v \in \mathbb{R}^{3}$. Let $\mathcal{P}_{ \pm}:=\operatorname{conv}(v \pm A)$ and $\mathcal{P}:=\mathcal{P}_{+} \cup \mathcal{P}_{-}$. Then $1-\langle\mathbf{u}, v\rangle$ does not appear in $\Omega(\mathbf{u})$, as

$$
F_{\mathcal{P}}(\mathbf{u})=F_{\mathcal{P}_{+}}(\mathbf{u})+F_{\mathcal{P}_{-}}(\mathbf{u})=K \frac{\sum_{1 \leq i<j \leq 3}\left\langle\mathbf{u}, a_{i}\right\rangle\left\langle\mathbf{u}, a_{j}\right\rangle+(1-\langle\mathbf{u}, v\rangle)^{2}}{\prod_{1 \leq i \leq 3}\left((1-\langle\mathbf{u}, v\rangle)^{2}-\left\langle\mathbf{u}, a_{i}\right\rangle^{2}\right)},
$$

where $K \neq 0$ is a real constant.

Now we introduce several finite-dimensional linear spaces related to a given finite spanning set $S \subset \mathbb{R}^{d}$. Let $\mathcal{P}(S)$ be the set of all generalized polytopes $\mathcal{P}$ whose sets $\mathcal{V}(\mathcal{P})$ of vertices are contained in $S$. For $\mathcal{P} \in \mathcal{P}(S)$, we denote by $\mu_{\mathcal{P}}$ its standard measure. (Obviously, $\mu_{\mathcal{P}}$ is supported on $\mathcal{P} \subseteq \operatorname{conv}(S)$.)

Denote by $\mathfrak{M}(S)$ the linear space of all signed measures, i.e. the linear span of all standard measures $\mu_{\mathcal{P}}$ for $\mathcal{P} \in \mathcal{P}(S)$. Let $\mathfrak{M}^{\Delta}(S) \subseteq \mathfrak{M}(S)$ be its subspace spanned by $\mu_{\Delta}$, for $\Delta \in \mathcal{P}(S)$ a $d$-dimensional simplex. (The space $\mathfrak{M}^{\Delta}(S)$ has earlier appeared in [2-4] in a somewhat different context.) We shall refer to elements of $\mathfrak{M}(S)$ as to polytopal measures with the vertex set $S$. The following conjecture was central to our study; it was pointed out to us that it follows from results in [13] after [19] was released (cf. Sect. 5.1 for a discussion). As well, at the same time authors of [1] started working 
on this question; their [1, Theorem 1], proved using a distinct from [13] set of ideas, implies the conjecture.

Conjecture 7 (Corollary to [1, Theorem 1]) For an arbitrary spanning set $S$ and any $\mathcal{P} \in \mathcal{P}(S)$, its standard measure $\mu_{\mathcal{P}}$ belongs to $\mathfrak{M}^{\Delta}(S)$. In other words, $\mathfrak{M}(S)=$ $\mathfrak{M}^{\Delta}(S)$.

By Remark 5, the above is non-trivial. In fact, [1] shows a stronger result, namely that the coefficients in a decomposition of $\mu_{c} P$ into a sum of $\mu_{\Delta}$ are integers, in particular resolving in the affirmative [19, Problem 3]. In view of this, we can make a stronger, "inclusion-exclusion"-like conjecture.

Conjecture 8 For an arbitrary spanning set $S$ and any $\mathcal{P} \in \mathcal{P}(S)$, its standard measure $\mu_{\mathcal{P}}$ can be decomposed as

$$
\mu_{\mathcal{P}}=\sum_{\Delta \in \mathcal{D}_{\mathcal{P}}} \sigma_{\Delta} \mu_{\Delta}, \quad \sigma_{\Delta}= \pm 1 \text { for all } \Delta,
$$

with $\mathcal{D}_{\mathcal{P}}$ a set of $d$-dimensional simplices in $\mathcal{P}(S)$.

Note that this holds true for $d=2$, as well as for any convex $\mathcal{P}$, with a stronger condition that all $\sigma_{\Delta}=1$.

While we did not have a proof of Conjecture 7 in its full generality, we have succeeded in proving it for a rather large class of spanning sets. Roughly speaking, the latter should be close to "generic". Specifically, given a finite spanning set $S \subset \mathbb{R}^{d}$, we say that $S$ is weakly non-degenerate if any $(d+2)$-tuple of points from $S$ is spanning. If $S$ satisfies the stronger condition that each $(d+1)$-subset of $S$ is spanning then we call the latter $S$ strongly non-degenerate.

Theorem 9 Conjecture 7 holds for any weakly non-degenerate finite set $S$.

Remark 7 Theorem 9 would imply Conjecture 7 if one could prove that the standard measure of an arbitrary generalized polytope $\mathcal{P}$ can be obtained as the limit of the standard measures of a 1-parameter family of generalized polytopes $\mathcal{P}(t)$ with $\mathcal{P}(0)=$ $\mathcal{P}$ such that for $t \neq 0$ the vertices of $\mathcal{P}(t)$ are weakly non-degenerate, and each vertex of $\mathcal{P}(t)$ tending to a vertex of $\mathcal{P}$ as $t \rightarrow 0$. We are unable to prove the existence of such deformations in general.

The key idea in the proof of Theorem 9 is to study the corresponding spaces of Fantappiè transformations of signed measures in $\mathfrak{M}(S)$. In particular, we are able to compute the corresponding dimensions ${ }^{1}$. In more detail, let $\mathfrak{F}(S)\left(\operatorname{resp} . \mathfrak{F}^{\Delta}(S)\right)$ be the linear space of Fantappiè transformations of signed measures in $\mathfrak{M}(S)\left(\right.$ resp. $\left.\mathfrak{M}^{\Delta}(S)\right)$. In other words, $\mathfrak{F}(S)$ (resp. $\mathfrak{F}^{\Delta}(S)$ ) is the space of normalized moment generating functions of signed measures in $\mathfrak{M}(S)$ (resp. $\left.\mathfrak{M}^{\Delta}(S)\right)$.

1 Note that presently we are not aware of a formula or a recipe for calculating the dimension of $\mathfrak{M}^{\Delta}(S)$ without the assumption of the theorem. (The manuscript [3] contains an algorithm constructing a basis of this space.) 
Since each compactly supported measure is uniquely determined by its complete set of moments, the map

$$
F_{\mu}: \mathfrak{M}(S) \rightarrow \mathfrak{F}(S),
$$

induced by the Fantappiè transformation is a linear isomorphism, cf. [5, Sect. 3.5].

Finally, given a spanning set $S=\left\{\mathbf{v}_{1}, \ldots, \mathbf{v}_{N}\right\} \subset \mathbb{R}^{d}$, denote by $\mathfrak{R a t}(S)$ the linear space of all rational functions with the denominator $\Phi_{S}(\mathbf{u})$ as in (1.12),

$$
\Phi_{S}(\mathbf{u})=\prod_{i=1}^{N}\left(1-\left\langle\mathbf{v}_{i}, \mathbf{u}\right\rangle\right),
$$

and with the numerator an arbitrary real (inhomogeneous) polynomial of degree at most $N-d-1$. Here the numerator and the denominator might have common factors.

Proposition $10 \mathfrak{F}^{\Delta}(S)$ coincides with $\mathfrak{R a t}(S)$ if and only if $S$ is strongly nondegenerate.

Corollary 11 If $S$ is strongly non-degenerate then $\mathfrak{M}^{\Delta}(S)=\mathfrak{M}(S)$.

Corollary 11 implies that for strongly non-degenerate $S$, the dimension of all these linear spaces equals $\left(\begin{array}{c}N-1 \\ d\end{array}\right)$. Note that Corollary 11 settles Theorem 9 for the strongly non-degenerate $S$.

Our final goal is to explicitly solve the following inverse moment problem.

Problem 1 Given a strongly non-degenerate spanning set $S \subset \mathbb{R}^{d},|S|=N$, find the unique polytopal measure in $\mathfrak{M}(S)$ with a given set of all moments up to order $N-d-1$.

We start with the following simple observation.

Lemma 12 Given an arbitrary spanning set $S \subset \mathbb{R}^{d},|S|=N$, and an arbitrary polynomial $T(\mathbf{u})$ of degree at most $N-d-1$, there exists a unique rational function $R(\mathbf{u})=P(\mathbf{u}) / \Phi_{S}(\mathbf{u})$ with Taylor polynomial of degree $N-d-1$ at the origin equal to $T(\mathbf{u})$. Namely, $P(\mathbf{u})=\left[T(\mathbf{u}) \Phi_{S}(\mathbf{u})\right]_{N-d-1}$, where $[\cdot]_{N-d-1}$ stands for the truncated polynomial with all monomials up to degree $N-d-1$.

For $S=\left\{\mathbf{v}_{1}, \ldots, \mathbf{v}_{N}\right\} \subset \mathbb{R}^{d}$ strongly non-degenerate, we give an explicit inversion formula determining the densities of an unknown polytopal measure having a given set of moments up to order $N-d-1$ on each simplex in a natural basis of $\mathfrak{M}^{\Delta}(S)$. In view of Lemma 12 we can assume that we are already given an arbitrary rational function $R(\mathbf{u})=P(\mathbf{u}) / \Phi_{S}(\mathbf{u})$, where $\operatorname{deg} P(\mathbf{u}) \leq N-d-1$, and we want to determine the densities of the required signed measure from $\mathfrak{M}(S)$ in terms of numerator $P(\mathbf{u})$.

From now on we shall choose the basis of $\mathfrak{M}^{\Delta}(S)$ consisting of the standard measures of all simplices containing the last vertex $\mathbf{v}_{N}$, see Lemma 16 below. Let $\mathfrak{L}=\left\{l_{1}, l_{2}, \ldots, l_{N-1}\right\}$ be the $(N-1)$-tuple of linear forms corresponding to vertices $\mathbf{v}_{1}, \mathbf{v}_{2}, \ldots, \mathbf{v}_{N-1}$, where $l_{i}(\mathbf{u})=1-\left\langle\mathbf{v}_{i}, \mathbf{u}\right\rangle$. Consider the linear span $V_{\mathfrak{L}}$ of all possible products of the form $l_{j_{1}} \cdot l_{j_{2}} \cdots l_{j_{N-d-1}}, 1 \leq j_{1}<j_{2}<\cdots<j_{N-d-1}$. There are $\left(\begin{array}{c}N-1 \\ d\end{array}\right)$ such products, and each of them is a polynomial of degree at most 
$N-d-1$. On the other hand, the dimension of the space $\operatorname{Pol}(N-d-1, d)$ of all (inhomogeneous) polynomials of degree at most $N-d-1$ in $d$ variables equals $\left(\begin{array}{c}N-1 \\ d\end{array}\right)$, as well.

Define the square matrix $\mathrm{Mat}_{S}$ of size $\left(\begin{array}{c}N-1 \\ d\end{array}\right)$ with entries being coefficients of the above products of linear forms with respect to the standard monomial basis in $\operatorname{Pol}(N-d-1, d)$. We assume that $M_{a t}$ acts on the space $V_{\mathfrak{L}}$ of column vectors.

Theorem 13 For an arbitrary strongly non-degenerate spanning set $S \subset \mathbb{R}^{d}$, $|S|=N$, the matrix Mats is invertible. Moreover, for a rational function $R(\mathbf{u})=$ $P(\mathbf{u}) / \Phi_{S}(\mathbf{u})$, where $P(\mathbf{u})$ is an arbitrary polynomial of degree $N-d-1$, there exists a unique measure $\mu_{R} \in \mathfrak{M}(S)$ with Fantappiè transform $R(\mathbf{u})$. Namely,

$$
\mu_{R}=\operatorname{Mat}_{S}^{-1}(P(\mathbf{u}))
$$

Remark 8 A detailed explanation of the meaning of (1.13) can be found in the proof of Theorem 13, see also Example 2 below. An explicit formula for the matrix $\mathrm{Mat}_{S}^{-1}$ is given in Lemma 19.

Recall that a spanning set $S$ is weakly non-degenerate if any $(d+2)$-tuple of its points is spanning. With minor changes, the above solution of the inverse moment problem can be adapted to this more general case. In order not to overload the introduction we refer the readers interested in this situation to Sect. 4 . The case of an arbitrary spanning set $S$, however, remains unsolved and offers several interesting challenges in matroid theory. We hope to return to it in the future.

It will be convenient to work with scaled volumes of simplices, which we call weights.

Definition 6 Given a signed measure $\mu$ in $\mathbb{R}^{d}$ and a $d$-dimensional simplex $\Delta \subset \mathbb{R}^{d}$, we define the weight $w_{\Delta}$ of $\Delta$ by the formula:

$$
w_{\Delta}=d ! \int_{\Delta} d \mu .
$$

In other words, the density $d_{\Delta}$ of the measure in question which should be placed at $\Delta$ equals

$$
d_{\Delta}=\frac{w_{\Delta}}{d ! \operatorname{Vol}(\Delta)}
$$

We finish the introduction by explicitly solving the above inverse problem for a concrete 5-tuple of points in $\mathbb{R}^{2}$.

Example 2 Set $S=\left\{\mathbf{v}_{1}, \mathbf{v}_{2}, \mathbf{v}_{3}, \mathbf{v}_{4}, \mathbf{v}_{5}\right\}$ where $\mathbf{v}_{1}=(1,0), \mathbf{v}_{2}=(2,1), \mathbf{v}_{3}=$ $(1,2), \mathbf{v}_{4}=(0,1), \mathbf{v}_{5}=(0,0)$. The corresponding set $\mathfrak{L}=\left\{l_{1}, l_{2}, l_{3}, l_{4}\right\}$ of linear forms is given by $l_{1}=1-u_{1}, l_{2}=1-2 u_{1}-u_{2}, l_{3}=1-u_{1}-2 u_{2}, l_{4}=1-u_{2}$. Additionally, $l_{5}=1$. We are considering the basis of $\mathfrak{M}^{\Delta}(S)$ consisting of (the standard measures of) 6 triangles containing $\mathbf{v}_{5}$. Therefore we need 6 quadratic forms obtained as pairwise products $l_{i} l_{j}, 1 \leq i<j \leq 4$. We get 


$$
\left\{\begin{array}{l}
l_{1} l_{2}=1-3 u_{1}-u_{2}+2 u_{1}^{2}+u_{1} u_{2} \\
l_{1} l_{3}=1-2 u_{1}-2 u_{2}+u_{1}^{2}+2 u_{1} u_{2} \\
l_{1} l_{4}=1-u_{1}-u_{2}+u_{1} u_{2} \\
l_{2} l_{3}=1-3 u_{1}-3 u_{2}+2 u_{1}^{2}+5 u_{1} u_{2}+2 u_{2} \\
l_{2} l_{4}=1-2 u_{1}-2 u_{2}+2 u_{1} u_{2}+u_{2}^{2} \\
l_{3} l_{4}=1-u_{1}-3 u_{2}+u_{1} u_{2}+2 u_{2}^{2}
\end{array}\right.
$$

Notice that $l_{1} l_{2}$ corresponds to triangle $\Delta_{345}, l_{1} l_{3}$ to $\Delta_{245}, l_{1} l_{3}$ to $\Delta_{245}, l_{1} l_{4}$ to $\Delta_{234}$, $l_{2} l_{3}$ to $\Delta_{145}, l_{2} l_{4}$ to $\Delta_{135}$, and $l_{3} l_{4}$ to $\Delta_{125}$. Ordering monomials spanning the space $\operatorname{Pol}(2,2)$ as $\left(1, u_{1}, u_{2}, u_{1}^{2}, u_{1} u_{2}, u_{2}^{2}\right)$, we get the $6 \times 6$-matrix Mats and its inverse $\mathrm{Mat}_{S}^{-1}$ as follows

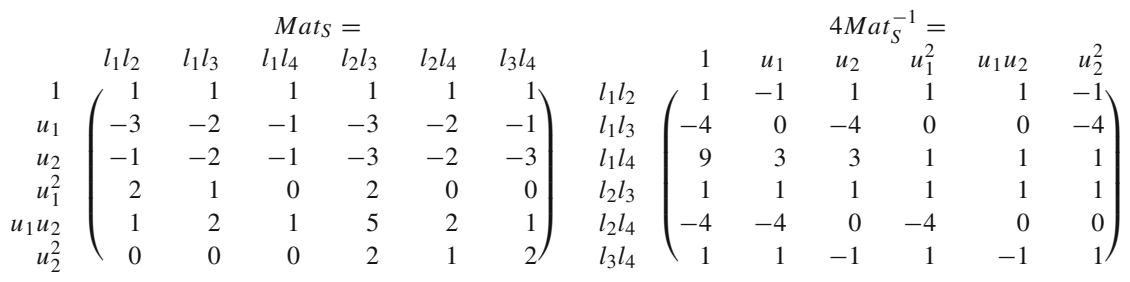

(For $\mathrm{T}_{\mathrm{E}} \mathrm{Xnical}$ reasons we give $4 M \mathrm{Mt}_{S}^{-1}$ above.) Thus, given an arbitrary rational function $R\left(u_{1}, u_{2}\right)=P\left(u_{1}, u_{2}\right) / \Phi_{S}\left(u_{1}, u_{2}\right)$ where $P\left(u_{1}, u_{2}\right)=a_{00}+a_{1,0} u_{1}+$ $a_{0,1} u_{2}+a_{2,0} u_{1}^{2}+a_{11} u_{1} u_{2}+a_{02} u_{2}^{2}$ is a polynomial of degree at most 2 and $\Phi_{S}\left(u_{1}, u_{2}\right)=$ $l_{1} l_{2} l_{3} l_{4} l_{5}$, we get

$$
\left\{\begin{array}{l}
w_{345}=\frac{1}{4}\left(a_{00}-a_{10}+a_{01}+a_{20}-a_{11}+a_{02}\right) \\
w_{245}=-a_{00}-a_{01}-a_{02} \\
w_{235}=\frac{1}{4}\left(9 a_{00}+3 a_{01}+3 a_{10}+a_{20}+a_{11}+a_{02}\right) \\
w_{145}=\frac{1}{4}\left(a_{00}+a_{01}+a_{10}+a_{20}+a_{11}+a_{02}\right) \\
w_{135}=-a_{00}-a_{10}-a_{20} \\
w_{125}=\frac{1}{4}\left(a_{00}+a_{10}-a_{01}+a_{20}-a_{11}+a_{02}\right)
\end{array}\right.
$$

where $w_{i j k}$ is the weight of the signed measure to be placed on $\Delta_{i j k}$, see (1.14).

To illustrate all steps of solution of our inverse moment problem, assume that we are looking for a polygonal measure with the vertex set $S$ and (ad hoc chosen) moments $m_{00}=1, m_{10}=2, m_{01}=3, m_{20}=4, m_{11}=5, m_{02}=6$. Then its normalized moment generating function $F_{\mu}(\mathbf{u})$ satisfies the relation

$$
\begin{aligned}
F_{\mu}(\mathbf{u})= & 1 \frac{2 !}{0 ! 0 !}+2 \frac{3 !}{1 ! 0 !} u_{1}+3 \frac{3 !}{0 ! 1 !} u_{2}+4 \frac{4 !}{2 ! 0 !} u_{1}^{2}+5 \frac{4 !}{1 ! 1 !} u_{1} u_{2} \\
& +6 \frac{4 !}{0 ! 2 !} u_{2}^{2}+\cdots=\frac{P\left(u_{1}, u_{2}\right)}{l_{1} l_{2} l_{3} l_{4} l_{5}},
\end{aligned}
$$




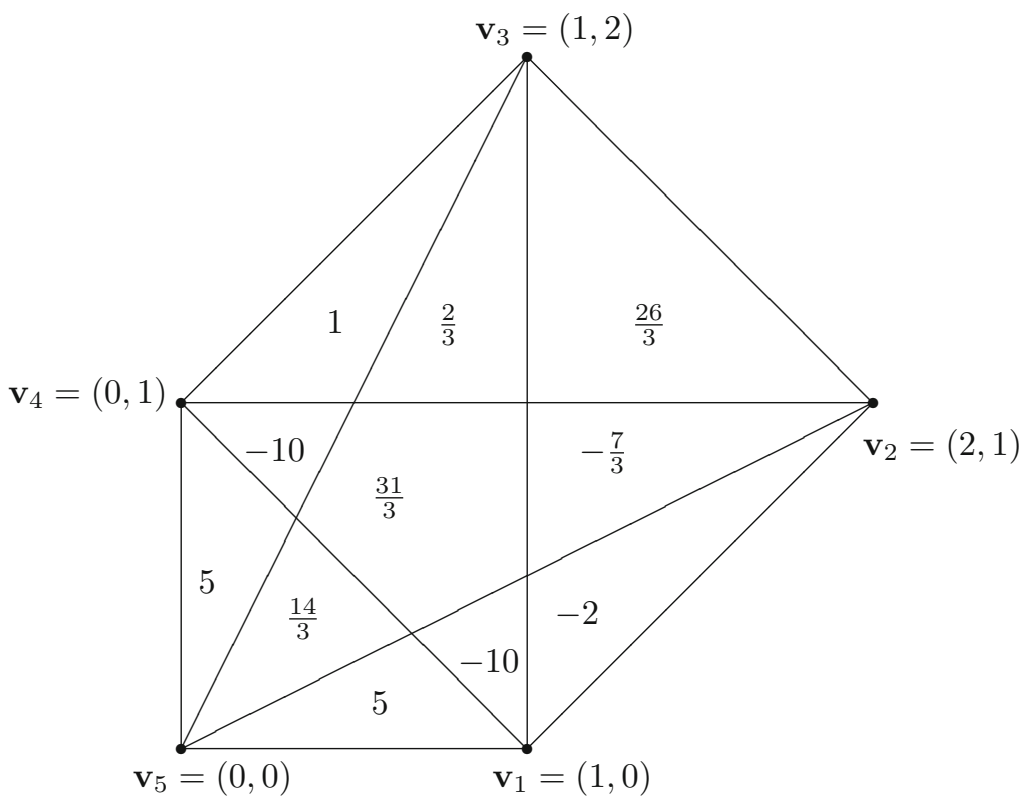

Fig. 2 Final measure in Example 2

where $P\left(u_{1}, u_{2}\right)$ is a (non-homogeneous) polynomial of at most second degree. Thus, truncating the product of the left-hand side and $l_{1} l_{2} l_{3} l_{4} l_{5}$ up to the second degree, we obtain

$$
P\left(u_{1}, u_{2}\right)=2+4 u_{1}+10 u_{2}+10 u_{1}^{2}+24 u_{1} u_{2}+10 u_{2}^{2},
$$

i.e. $a_{00}=2, a_{10}=4, a_{01}=10, a_{20}=10, a_{11}=24, a_{02}=10$. Thus $w_{345}=1, w_{245}=-22, w_{235}=26, w_{145}=15, w_{135}=-16, w_{125}=-2$. The areas of the corresponding triangles are equal to: $\operatorname{Area}\left(\Delta_{345}\right)=\frac{1}{2} ; \operatorname{Area}\left(\Delta_{245}\right)=$ $1 ; \operatorname{Area}\left(\Delta_{235}\right)=\frac{3}{2} ; \operatorname{Area}\left(\Delta_{145}\right)=\frac{1}{2} ; \operatorname{Area}\left(\Delta_{135}\right)=1 ; \operatorname{Area}\left(\Delta_{125}\right)=\frac{1}{2}$. This implies that the densities of the measure of the corresponding triangles are equal to $d_{345}=1, d_{245}=-11, d_{235}=\frac{26}{3}, d_{145}=15, d_{135}=-8, d_{125}=-2$. To obtain the final densities in the convex hull $\operatorname{conv}(S)$ of $S$, one has to decompose $\operatorname{conv}(S)$ into domains obtained by removing from $\operatorname{conv}(S)$ the set of all hyperplanes spanned by vertices in $S$. For each such domain, we should add up the densities of all basic simplices containing this domain. The resulting measure is shown in Fig. 2.

Remark 9 Domains into which the convex hull $\operatorname{conv}(S)$ is cut by the hyperplanes spanned by $S$ were introduced in [2] where they were called chambers. The incidence matrix of the simplices spanned by $S$ and those chambers was studied in some detail in $[3,4]$. This matrix allows to formalize the last step of construction of the above polygonal measure, where information on the densities of the simplices is transformed into information on the densities of the chambers. But, in general, already the number 
of chambers is a complicated invariant of the set $S$. It seems that the general problem of constructing the set of chambers and the corresponding incidence matrix in terms of a given $S$ is quite non-trivial.

\section{Proving results on convex polytopes}

Following Brion-Lawrence-Khovanskii-Pukhlikov-Barvinok, see [7,9,20,22], we define for each vector $\mathbf{z} \in \mathbb{R}^{d}$, the $j$-th axial moment $\mu_{j}(\mathbf{z})$ of a simple convex polytope $\mathcal{P}$ with respect to $\mathbf{z}$ as

$$
\mu_{j}(\mathbf{z})=\int_{\mathcal{P}}\langle\mathbf{x}, \mathbf{z}\rangle^{j} d \mathbf{x}
$$

We will use the following important statement, cf. e.g. [9, Theorem 10.5].

Theorem 14 The moment $\mu_{j}(\mathbf{z})$ satisfies

$$
\mu_{j}(\mathbf{z})=\frac{(-1)^{d} j !}{(j+d) !} \sum_{\mathbf{v} \in \mathcal{V}}\langle\mathbf{v}, \mathbf{z}\rangle^{j+d} D_{\mathbf{v}}(\mathbf{z}),
$$

where $D_{\mathbf{v}}(\mathbf{z}):=\frac{\left|\operatorname{det} K_{\mathbf{v}}\right|}{\prod_{j=1}^{d}\left\langle w_{j}(\mathbf{v}), \mathbf{z}\right\rangle}$, and $\mathbf{z}$ is an arbitrary vector for which the products $\prod_{j=1}^{d}\left\langle w_{j}(\mathbf{v}), \mathbf{z}\right\rangle, \mathbf{v} \in \mathcal{V}$, do not vanish. Moreover, the following identities hold:

$$
\sum_{\mathbf{v} \in \mathcal{V}}\langle\mathbf{v}, \mathbf{z}\rangle^{j} D_{\mathbf{v}}(\mathbf{z})=0, \quad \text { for } 0 \leq j \leq d-1
$$

Proof of Theorem 2 To prove (1.8), consider the generating function

$$
\Psi_{\mathbf{z}}(u)=\sum_{j=0}^{\infty} \frac{(j+d) !}{j !} \mu_{j}(\mathbf{z}) u^{j}
$$

where $u \in \mathbb{R}$. Formula (2.1) implies that $\Psi_{\mathbf{z}}(u)$ is rational. Indeed,

$$
\begin{aligned}
\Psi_{\mathbf{z}}(u) & =\sum_{j=0}^{\infty}(-1)^{d} \sum_{\mathbf{v} \in \mathcal{V}}\langle\mathbf{v}, \mathbf{z}\rangle^{j+d} \frac{\left|\operatorname{det} K_{\mathbf{v}}\right| u^{j}}{\prod_{k=1}^{d}\left\langle w_{k}(\mathbf{v}), \mathbf{z}\right\rangle} \\
& =(-1)^{d} \sum_{\mathbf{v} \in \mathcal{V}} \frac{\langle\mathbf{v}, \mathbf{z}\rangle^{d}\left|\operatorname{det} K_{\mathbf{v}}\right|}{\prod_{k=1}^{d}\left\langle w_{k}(\mathbf{v}), \mathbf{z}\right\rangle} \sum_{j=0}^{\infty}\langle\mathbf{v}, \mathbf{z}\rangle^{j} u^{j}=\sum_{\mathbf{v} \in \mathcal{V}} \frac{\langle\mathbf{v}, \mathbf{u}\rangle^{d}\left|\operatorname{det} K_{\mathbf{v}}\right|}{\prod_{k=1}^{d}\left\langle w_{k}(\mathbf{v}), \mathbf{u}\right\rangle} \cdot \frac{(-1)^{d}}{1-\langle\mathbf{v}, \mathbf{u}\rangle},
\end{aligned}
$$


where $\mathbf{u}=u \mathbf{z}$. On the other hand, using the multinomial coefficients $\left(\begin{array}{c}|J| \\ J\end{array}\right)=\frac{|J| !}{j_{1} ! \ldots j_{d} !}$ of multiindices $J=\left(j_{1}, \ldots, j_{d}\right) \vdash|J|$, one gets

$$
\int_{\mathcal{P}}\langle\mathbf{x}, \mathbf{z}\rangle^{j} d \mathbf{x}=\int_{\mathcal{P}}\left(\sum_{i=1}^{d} x_{i} z_{i}\right)^{j} d \mathbf{x}=\sum_{J \vdash j}\left(\begin{array}{l}
j \\
J
\end{array}\right) \mathbf{z}^{J} \int_{\mathcal{P}} \mathbf{x}^{J} d \mathbf{x}=\sum_{J \vdash j}\left(\begin{array}{l}
j \\
J
\end{array}\right) \mathbf{z}^{J} m_{J}(\mathcal{P}),
$$

where $m_{J}(\mathcal{P})=m_{J}\left(\mu_{\mathcal{P}}\right)$. Therefore,

$$
\begin{aligned}
F_{\mathcal{P}}(u \mathbf{z}) & :=F_{\mathcal{P}}\left(u z_{1}, \ldots, u z_{d}\right):=\sum_{j=0}^{\infty} \sum_{\left(j_{1}, \ldots, j_{d}\right) \vdash j} \frac{(j+d) !}{j_{1} ! \ldots j_{d} !} m_{j_{1}, \ldots, j_{d}}\left(u z_{1}\right)^{j_{1}} \cdots\left(u z_{d}\right)^{j_{d}} \\
& =\sum_{j=0}^{\infty} \frac{(j+d) !}{j !}\left[\sum_{J:=\left(j_{1}, \ldots, j_{d}\right) \vdash j}\left(\begin{array}{l}
j \\
J
\end{array}\right) m_{J}(\mathcal{P}) \mathbf{z}^{J}\right] u^{j} \\
& =\sum_{j=0}^{\infty} \frac{(j+d) !}{j !} \mu_{j}(\mathbf{z}) u^{j}=\Psi_{\mathbf{z}}(u),
\end{aligned}
$$

and (1.8) follows.

In view of relations (2.2), the right-hand side of (1.8) can be rewritten as (1.9). Indeed, writing $(1-\langle\mathbf{v}, \mathbf{u}\rangle)^{-1}=\sum_{j=0}^{\infty}\langle\mathbf{v}, \mathbf{u}\rangle^{j}$ and expanding (1.8) with respect to $j$ th powers of $\langle\mathbf{v}, \mathbf{u}\rangle$, we see that (2.2) implies that for $j<d$ the sum of all terms $\langle\mathbf{v}, \mathbf{u}\rangle^{j}$ vanishes.

Proof of Corollary 3 Let $\mathcal{V}=\left(\mathbf{v}_{0}, \mathbf{v}_{1}, \ldots, \mathbf{v}_{d}\right)$. Then for each $j \neq i$, we have $w_{j}\left(\mathbf{v}_{i}\right)=\mathbf{v}_{j}-\mathbf{v}_{i}$. Hence $\left|\operatorname{det} K_{\mathbf{v}_{i}}\right|$ does not depend upon $i$ and equals $d ! \operatorname{Vol}(\Delta)$. The right-hand side of (1.8) becomes

$$
\begin{aligned}
& (-1)^{d} d ! \operatorname{Vol}(\Delta) \sum_{i=0}^{d} \frac{\left\langle\mathbf{v}_{i}, \mathbf{u}\right\rangle^{d}}{\prod_{j=1}^{d}\left\langle w_{j}\left(\mathbf{v}_{i}\right), \mathbf{u}\right\rangle} \cdot \frac{1}{1-\left\langle\mathbf{v}_{i}, \mathbf{u}\right\rangle} \\
& =(-1)^{d} d ! \operatorname{Vol}(\Delta) \sum_{i=0}^{d} \frac{\left\langle\mathbf{v}_{i}, \mathbf{u}\right\rangle^{d}}{\prod_{j \neq i}\left\langle\mathbf{v}_{j}-\mathbf{v}_{i}, \mathbf{u}\right\rangle} \cdot \frac{1}{1-\left\langle\mathbf{v}_{i}, \mathbf{u}\right\rangle} \\
& =(-1)^{d} d ! \operatorname{Vol}(\Delta) \sum_{i=0}^{d} \frac{\zeta_{i}^{d}}{\prod_{j \neq i}\left(\zeta_{j}-\zeta_{i}\right)} \cdot \frac{1}{1-\zeta_{i}},
\end{aligned}
$$

where $\zeta_{i}=\left\langle\mathbf{v}_{i}, \mathbf{u}\right\rangle$. Computing the common denominator of the latter, we obtain

$$
F_{\Delta}(\mathbf{u})=\frac{(-1)^{d} d ! \operatorname{Vol}(\Delta)}{\prod_{i=0}^{d}\left(1-\zeta_{i}\right)} \frac{\sum_{i=0}^{d}\left[\prod_{k>l, k \neq i \neq l}\left(\zeta_{k}-\zeta_{l}\right) \prod_{j \neq i}\left(1-\zeta_{j}\right)\right](-1)^{i} \zeta_{i}^{d}}{\prod_{s>t}\left(\zeta_{s}-\zeta_{t}\right)} .
$$


It is convenient to introduce one more linear form $\zeta_{d+1}:=1$, so that the last expression reads as

$$
F_{\Delta}(\mathbf{u})=(-1)^{d} d ! \operatorname{Vol}(\Delta) \frac{\sum_{\substack{i=0 \\ d}}\left[\prod_{\substack{d+1 \geq k>l \geq 0 \\ k \neq i \neq l}},\left(\zeta_{k}-\zeta_{l}\right)\right](-1)^{i} \zeta_{i}^{d}}{\prod_{d+1 \geq s>t \geq 0}\left(\zeta_{s}-\zeta_{t}\right)} .
$$

To complete the proof, we notice that

$$
\begin{aligned}
& 0=\operatorname{det}\left(\begin{array}{ccccc}
1 & 1 & \ldots & 1 & 1 \\
\zeta_{0} & \zeta_{1} & \ldots & \zeta_{d} & 1 \\
\zeta_{0}^{2} & \zeta_{1}^{2} & \ldots & \zeta_{d}^{2} & 1 \\
\ldots & & & & \\
\zeta_{0}^{d} & \zeta_{1}^{d} & \ldots & \zeta_{d}^{d} & 1 \\
\zeta_{0}^{d} & \zeta_{1}^{d} & \ldots & \zeta_{d}^{d} & 1
\end{array}\right)=1 \cdot \operatorname{det}\left(\begin{array}{cccc}
1 & 1 & \ldots & 1 \\
\zeta_{0} & \zeta_{1} & \ldots & \zeta_{d} \\
\zeta_{0}^{2} & \zeta_{1}^{2} & \ldots & \zeta_{d}^{2} \\
\ldots & & & \\
\zeta_{0}^{d} & \zeta_{1}^{d} & \ldots & \zeta_{d}^{d}
\end{array}\right) \\
& +(-1)^{d+1} \sum_{i=0}^{d} \zeta_{i}^{d}(-1)^{i} \cdot \operatorname{det}\left(\begin{array}{cccccc}
1 & \ldots & 1 & 1 & \ldots & 1 \\
\zeta_{0} & \ldots & \zeta_{i-1} & \zeta_{i+1} & \ldots & \zeta_{d+1} \\
\zeta_{0}^{2} & \ldots & \zeta_{i-1}^{2} & \zeta_{i+1}^{2} & \ldots & \zeta_{d+1}^{2} \\
\ldots & & & & & \\
\zeta_{0}^{d} & \ldots & \zeta_{i-1}^{d} & \zeta_{i+1}^{d} & \ldots & \zeta_{d+1}^{d}
\end{array}\right) \\
& =\prod_{d \geq k>l \geq 0}\left(\zeta_{k}-\zeta_{l}\right)+(-1)^{d+1} \sum_{i=0}^{d} \zeta_{i}^{d}(-1)^{i} \cdot\left[\prod_{\substack{d \geq k>l \geq 0, k \neq i \neq l}}\left(\zeta_{k}-\zeta_{l}\right)\right] \text {. }
\end{aligned}
$$

Indeed, the first matrix has two identical rows and thus vanishing determinant, which we expand with respect to the last row. The last equality is the standard formula for the Vandermonde determinant. Thus we have

$$
\sum_{i=0}^{d} \zeta_{i}^{d}(-1)^{i} \cdot\left[\prod_{\substack{d+1 \geq k>l \geq 0, k \neq i \neq l}}\left(\zeta_{k}-\zeta_{l}\right)\right]=(-1)^{d} \prod_{d \geq k>l \geq 0}\left(\zeta_{k}-\zeta_{l}\right)
$$

Now we plug this formula into (2.3) and get

$$
F_{\Delta}(\mathbf{u})=d ! \operatorname{Vol}(\Delta) \frac{1}{\prod_{t=0}^{d}\left(1-\zeta_{t}\right)}=\frac{d ! \operatorname{Vol}(\Delta)}{\prod_{\mathbf{v} \in \mathcal{V}}(1-\langle\mathbf{v}, \mathbf{u}\rangle)} .
$$

Lemma 15 Let $\mathbf{u}=\left(u_{1}, \ldots, u_{d}\right)$ and $\mathbf{x}=\left(x_{1}, \ldots, x_{d}\right)$ be formal variables, and $\ell \in \mathbb{R}$. Then

$$
\left(\sum_{k} u_{k} \frac{\partial}{\partial u_{k}}+\ell\right) \circ(1-\langle\mathbf{x}, \mathbf{u}\rangle)^{-\ell}=\ell(1-\langle\mathbf{x}, \mathbf{u}\rangle)^{-\ell-1} .
$$


Proof Note that $u_{k} \frac{\partial}{\partial u_{k}} \circ(1-\langle\mathbf{x}, \mathbf{u}\rangle)^{-\ell}=x_{k} u_{k} \ell(1-\langle\mathbf{x}, \mathbf{u}\rangle)^{-\ell-1}$. Thus

$$
\begin{aligned}
\left(\sum_{k} u_{k} \frac{\partial}{\partial u_{k}}+\ell\right) \circ(1-\langle\mathbf{x}, \mathbf{u}\rangle)^{-\ell} & =\ell(1-\langle\mathbf{x}, \mathbf{u}\rangle)^{-\ell-1}\langle\mathbf{x}, \mathbf{u}\rangle+\ell(1-\langle\mathbf{x}, \mathbf{u}\rangle)^{-\ell} \\
& =\ell(1-\langle\mathbf{x}, \mathbf{u}\rangle)^{-\ell-1}
\end{aligned}
$$

Proof of (1.3) For a $d$-variate polynomial $g(\mathbf{z})$, we denote by $g\left(\mathbf{u} \frac{\partial}{\partial \mathbf{u}}\right)$ the differential operator $g\left(u_{1} \frac{\partial}{\partial u_{1}}, \ldots, u_{d} \frac{\partial}{\partial u_{d}}\right)$. We use the identity

$$
g\left(\mathbf{u} \frac{\partial}{\partial \mathbf{u}}\right) \circ \sum_{I} a_{I} \mathbf{x}^{I} \mathbf{u}^{I}=\sum_{I \geq 0} g(I) a_{I} \mathbf{x}^{I} \mathbf{u}^{I}
$$

which holds for any formal $d$-variate power series $\sum_{I} a_{I} \mathbf{x}^{I} \mathbf{u}^{I}$ and any $d$-variate polynomial $g(\mathbf{z})$. (It can be easily verified for monomial $g(\mathbf{z})$ and then extended by linearity.) Setting $h(\mathbf{z}):=\prod_{\ell=1}^{d}\left(\sum_{k=1}^{d} z_{k}+\ell\right)$, notice that $h(I)=(|I|+1)(|I|+$ 2) $\cdots(|I|+d)$. Now using (2.5) together with the obvious identity:

$$
(1-\langle\mathbf{x}, \mathbf{u}\rangle)^{-1}=\sum_{I \geq 0}\left(\begin{array}{c}
|I| \\
I
\end{array}\right) \mathbf{x}^{I} \mathbf{u}^{I}
$$

one obtains

$$
\begin{aligned}
F_{\mu}(\mathbf{u}) & :=\sum_{I \geq 0}\left(\begin{array}{c}
|I|+d \\
I
\end{array}\right) m_{I}(\mu) \mathbf{u}^{I}=\sum_{I \geq 0} h(I)\left(\begin{array}{c}
|I| \\
I
\end{array}\right) m_{I}(\mu) \mathbf{u}^{I} \\
& =\int_{\mathbb{R}^{d}} \sum_{I \geq 0} h(I)\left(\begin{array}{c}
|I| \\
I
\end{array}\right) \mathbf{x}^{I} \mathbf{u}^{I} d \mu(\mathbf{x})=\int_{\mathbb{R}^{d}} h\left(\mathbf{u} \frac{\partial}{\partial \mathbf{u}}\right) \circ \sum_{I \geq 0}\left(\begin{array}{c}
|I| \\
I
\end{array}\right) \mathbf{x}^{I} \mathbf{u}^{I} d \mu(\mathbf{x}) \\
& =\int_{\mathbb{R}^{d}} h\left(\mathbf{u} \frac{\partial}{\partial \mathbf{u}}\right) \circ \frac{d \mu(\mathbf{x})}{1-\langle\mathbf{x}, \mathbf{u}\rangle}=\int_{\mathbb{R}^{d}} \frac{d ! d \mu(\mathbf{x})}{(1-\langle\mathbf{x}, \mathbf{u}\rangle)^{d+1}},
\end{aligned}
$$

where in the final derivation we repeatedly made use of (2.4), for $1 \leq \ell \leq d$.

Remark 10 Another point of view on (1.3) is that it is the result of the application of the differential operator $g\left(\mathbf{u} \frac{\partial}{\partial \mathbf{u}}\right)$ to the integral transformation $\int_{\mathbb{R}^{d}} \frac{d \mu(\mathbf{x})}{1-\langle\mathbf{x}, \mathbf{u}\rangle}$ of the measure $\mu$ (also known as the Fantappiè transform of $\mu$ ); see e.g. [23].

In [26] a similar idea was applied to the harmonic polygonal measures in the plane.

Proof of Theorem 1 Assume first that $\rho\left(x_{1}, \ldots, x_{d}\right)=\mathbf{x}^{K}=x_{1}^{k_{1}} \cdots x_{d}^{k_{d}}$ is a monomial and consider

$$
\rho\left(\frac{\partial}{\partial \mathbf{u}}\right) \circ F_{\mu}(\mathbf{u})=\frac{\partial^{|K|}}{\partial u_{1}^{k_{1}} \cdots \partial u_{d}^{k_{d}}} \circ \sum_{I=\left(i_{1}, \ldots, i_{d}\right) \geq 0} \frac{(|I|+d) !}{i_{1} ! \cdots i_{d} !} m_{I}(\mu) \mathbf{u}^{I} .
$$


One gets

$$
\begin{aligned}
\frac{\partial^{|K|}}{\partial \mathbf{u}^{K}} \circ F_{\mu}(\mathbf{u}) & =\sum_{I=\left(i_{1}, \ldots, i_{d}\right) \geq 0}\left(\frac{(|I|+|K|+d) !}{\prod_{j=1}^{d}\left(i_{j}+k_{j}\right) !} \prod_{j=1}^{d} \frac{\left(i_{j}+k_{j}\right) !}{i_{j} !}\right) m_{I+K}(\mu) \mathbf{u}^{I} \\
& =\sum_{I=\left(i_{1}, \ldots, i_{d}\right) \geq 0} \frac{(|I|+d+|K|) !}{\prod_{j=1}^{d} i_{j} !} m_{I}\left(\mathbf{x}^{K} \mu\right) \mathbf{u}^{I} .
\end{aligned}
$$

Observe that the normalizing coefficients of $m_{I}\left(\mathbf{x}^{K} \mu\right)$ in the latter expression depend only on $I$ and $|K|$ but not on particular entries of $K$. Therefore, for an arbitrary homogeneous $\rho$ of degree $\delta$, one gets by additivity

$$
\rho\left(\frac{\partial}{\partial \mathbf{u}}\right) \circ F_{\mu}(\mathbf{u})=\sum_{I=\left(i_{1}, \ldots, i_{d}\right) \geq 0} \frac{(|I|+d+\delta) !}{\prod_{j=1}^{d} i_{j} !} m_{I}(\rho \mu) .
$$

This shows (1.6). Repeated application of (2.4), for $d+1 \leq \ell \leq d+\delta$, to the integral representation (1.3), respectively, to the representation (1.2), of $F_{\rho \mu}(\mathbf{u})$ implies (1.7), respectively, (1.5).

\section{Inverse moment problem for strongly non-degenerate $S$}

Proof of Lemma 5 We prove first that the tangent cone at any non-vertex allows a decomposition into convex polytopal cones each having a translation-invariant direction.

Let $\mathbf{v}$ be a point in $\mathcal{P}$ which is not a vertex. Then there is a dissection $\mathcal{T}$ of $\mathcal{P}$ such that $\mathbf{v}$ is not a vertex of any simplex of $\mathcal{T}$. Let $U$ be the set of simplices $S_{u}$ of $\mathcal{T}$ with closures containing $\mathbf{v}$. Take the dissection of the tangent cone $T_{v}(\mathcal{P})$ into the tangent cones to simplices from $U, T_{\mathbf{v}}(\mathcal{P})=\cup_{u \in U} T_{\mathbf{v}}\left(S_{u}\right)$. Clearly, every subcone $T_{v}\left(S_{u}\right)$ contains a translation-invariant direction (any direction parallel to the minimal face containing $\mathbf{v}$ ).

Vice versa, to prove the converse implication, let us take a dissection of the tangent cone $T_{\mathbf{v}}(\mathcal{P})$ into a disjoint union of convex polytopal cones $Q_{1}, \ldots, Q_{k}$. By definition of the tangent cone and since $\mathcal{P}$ can be represented as a finite union of simplices, we obtain that any sufficiently small neighborhood of $\mathbf{v}$ in the tangent cone $T_{\mathbf{v}}(\mathcal{P})$ is a neighborhood of $\mathbf{v}$ in the entire $\mathcal{P}$. Consider the parallelepiped $\operatorname{Box}_{\varepsilon}$ centered at $\mathbf{v}$ that is the $\varepsilon$-ball centered at $\mathbf{v}$, in the $L_{1}$-norm. Note that each convex polytopal set $Q_{i} \cap \operatorname{Box}_{\varepsilon}$ can be decomposed into a union of simplices that do not contain $\mathbf{v}$ as a vertex.

Further notice that the set $\mathcal{P} \backslash \mathrm{Box}_{\varepsilon}$ can be represented as a finite disjoint union of simplices, since $\mathrm{Box}_{\mathcal{E}}$ is the intersection of a finite number of half-spaces and $\mathcal{P}$ is a disjoint union of simplices. Clearly, every simplex in this union should not have $\mathbf{v}$ as a vertex. Now combining the dissections of each $Q_{i} \cap \operatorname{Box}_{\varepsilon}$ and $\mathcal{P} \backslash \operatorname{Box}_{\varepsilon}$ we obtain the required dissection of $\mathcal{P}$. 
Proof of Proposition 6 We begin by considering the case $\rho \equiv 1$. Let $\mathcal{T}$ be a dissection of $\mathcal{P}$ with vertices $\mathcal{V}(\mathcal{T})$. Corollary 3 implies that $F_{\mathcal{P}}(\mathbf{u})$ has a denominator dividing $g_{\mathcal{T}}(\mathbf{u})=\prod_{\mathbf{v} \in \mathcal{V}(\mathcal{T})}(1-\langle\mathbf{v}, \mathbf{u}\rangle)$. Take $\mathbf{v}_{1} \in \mathcal{V}(\mathcal{T}) \backslash \mathcal{V}(\mathcal{P})$. Then there exists another dissection $\mathcal{T}^{\prime}$ such that $\mathbf{v}_{1} \notin \mathcal{V}\left(\mathcal{T}^{\prime}\right)$. Expressing $F_{\mathcal{P}}(\mathbf{u})$ as ratios of polynomials, we have

$$
F_{\mathcal{P}}(\mathbf{u})=\frac{f_{\mathcal{T}}(\mathbf{u})}{h_{\mathcal{T}}(\mathbf{u})\left(1-\left\langle\mathbf{v}_{1}, \mathbf{u}\right\rangle\right)}=\frac{f_{\mathcal{T}^{\prime}}(\mathbf{u})}{g_{\mathcal{T}^{\prime}}(\mathbf{u})}, \quad \text { where } g_{\mathcal{T}}(\mathbf{u})=h_{\mathcal{T}}(\mathbf{u})\left(1-\left\langle\mathbf{v}_{1}, \mathbf{u}\right\rangle\right)
$$

Here $g_{\mathcal{T}}$, is not divisible by $1-\left\langle\mathbf{v}_{1}, \mathbf{u}\right\rangle$, by the choice of $\mathcal{T}^{\prime}$. Thus $f_{\mathcal{T}}$ is divisible by $1-\left\langle\mathbf{v}_{1}, \mathbf{u}\right\rangle$, and can be canceled out in the expression for $F_{\mathcal{P}}(\mathbf{u})$.

The case of arbitrary homogeneous $\rho$ follows immediately by applying Theorem 1 to the already covered case $\rho \equiv 1$.

Proof of Proposition 10 First we show that for an arbitrary finite spanning set $S \subset \mathbb{R}^{d}$, the space $\mathfrak{M}^{\Delta}(S)$ has a basis of $d$-dimensional simplices containing a fixed vertex $\mathbf{v} \in S$. In particular, the set of all $d$-dimensional simplices containing $\mathbf{v}$ spans $\mathfrak{M}^{\Delta}(S)$ but is not necessarily a basis. Consequently, their Fantappiè transformations span $\mathfrak{F}^{\Delta}(S)$. The following result is formulated as Theorem 4.2 of [3] and in a different form in [2]. (We omit the proof of this statement here.)

Given two points $p$ and $q$ and a set $M$ in $\mathbb{R}^{d}$, we say that $q$ is visible from $p$ with respect to $M$ if the line segment $p q$ is disjoint from $M$.

Lemma 16 Given ad-dimensional simplex $\sigma \subset \mathbb{R}^{d}$, denote by $\mathcal{V}(\sigma)$ the set of vertices of $\sigma$. Let $\sigma^{0}$ be the interior of $\sigma$. Let $p$ be any point in $\mathbb{R}^{d}$ and let $Q^{+}$(resp. $Q^{-}$) be the set of all $(d-1)$-dimensional faces of $\sigma$ which are visible (resp. not visible) from $p$ with respect to $\sigma^{0}$. Then the standard measures of all d-dimensional simplices with vertices in $\mathcal{V}(\sigma) \cup\{p\}$ satisfy

$$
\mu_{\sigma}=\sum_{\sigma_{i} \in Q^{+}} \mu_{\sigma_{i}, p}-\sum_{\sigma_{i} \in Q^{-}} \mu_{\sigma_{i}, p}
$$

where $\mu_{\sigma_{i}, p}$ is the standard measure of the d-dimensional simplex spanned by the vertices of $\sigma_{i}$ and the point $p$.

Remark 11 If $\sigma_{i, p}$ is a degenerate simplex, i.e., $p$ lies in the hyperplane spanned by $\sigma_{i}$, we simply exclude the corresponding term $\mu_{\sigma_{i, p}}$ from the above formula.

To prove Proposition 10, we need to show that $\mathfrak{F}^{\Delta}(S)$ coincides with $\mathfrak{R a t}(S)$ if and only if $S$ is strongly non-degenerate. Indeed, $\mathfrak{F}^{\Delta}(S) \subseteq \mathfrak{R a t}(S)$ for an arbitrary spanning $S$, by Proposition 6. The Fantappiè transform $F_{\mu}: \mathfrak{M}^{\Delta}(S) \rightarrow \mathfrak{F}^{\Delta}(S)$ is a linear isomorphism which implies that $\operatorname{dim} \mathfrak{M}^{\Delta}(S)=\operatorname{dim} \mathfrak{F}^{\Delta}(S)$. By Lemma 16, the space $\mathfrak{M}^{\Delta}(S)$ is spanned by the standard measures $\mu_{\Delta}$ of the set $\mathcal{B}_{i}$ of all $d$-dimensional simplices containing the fixed vertex $\mathbf{v}_{i}$. Let us fix the vertex $\mathbf{v}_{N}$ and consider the set $\mathcal{B}_{N}$. For $S$ strongly non-degenerate, the cardinality of $\mathcal{B}_{N}$ equals $\left(\begin{array}{c}N-1 \\ d\end{array}\right)$.

Now we show that $\mathfrak{F}^{\Delta}(S)=\mathfrak{R a t}(S)$, where $\mathfrak{R a t}(S)$ has the dimension $\left(\begin{array}{c}N-1 \\ d\end{array}\right)$, as it is isomorphic to the space $\operatorname{Pol}(N-d-1, d)$ of all $d$-variate polynomials of degree 
at most $N-d-1$. This would immediately imply that the standard measures of simplices in $\mathcal{B}_{N}$ are linearly independent.

Lemma 17 If $S$ is strongly non-degenerate, then $\mathfrak{F}^{\Delta}(S)=\mathfrak{R a t}(S)$.

Proof We recall that $\mathfrak{F}^{\Delta}(S)$ comprises all linear combinations of the rational functions

$$
\frac{d ! \operatorname{Vol}\left(\mathbf{v}_{i_{1}}, \ldots, \mathbf{v}_{i_{d}}, \mathbf{v}_{N}\right)}{\left(1-\left\langle\mathbf{v}_{i_{1}}, \mathbf{u}\right\rangle\right) \cdots \cdot\left(1-\left\langle\mathbf{v}_{i_{d}}, \mathbf{u}\right\rangle\right) \cdot\left(1-\left\langle\mathbf{v}_{N}, \mathbf{u}\right\rangle\right)} .
$$

For each term $1-\left\langle\mathbf{v}_{i}, \mathbf{u}\right\rangle$, we consider a (homogeneous) linear form $l_{i}\left(u_{0}, \mathbf{u}\right)=$ $u_{0}-\left\langle\mathbf{v}_{i}, \mathbf{u}\right\rangle$ in $d+1$ variables $u_{0}, \ldots, u_{d}$. Set $n=N-1$ where $n \geq d+1$. For the $n$ tuple $\mathfrak{L}=\left\{l_{1}, l_{2}, \ldots, l_{n}\right\}$ of linear $(d+1)$-variate forms, let $V_{\mathfrak{L}}$ be the linear span of all possible products of the form $l_{i_{1}} l_{i_{2}} \ldots l_{i_{n-d}}, 1 \leq i_{1}<i_{2}<\cdots<i_{n-d} \leq n$. Observe that $V_{\mathfrak{L}}$ is the space of all numerators that one can obtain in $\mathfrak{F}^{\Delta}(S)$. We need to show that $V_{\mathfrak{L}}$ contains $H \operatorname{Pol} y(n-d, d+1)$, the space of all $(d+1)$-variate homogeneous polynomials of degree $n-d$. Recall that any $d+1$-tuple of linear forms $l_{i_{1}}, \ldots, l_{i_{d+1}}$ is linearly independent due to the strong degeneracy assumption. Thus we can express each single variable $u_{0}, \ldots, u_{d}$ as a linear combination of these forms. Since $V_{\mathfrak{L}}$ contains all products $l_{i_{1}} l_{i_{2}} \ldots l_{i_{n-d-1}} l_{j}$, where $j \in\{1, \ldots, n\} \backslash\left\{i_{1}, \ldots, i_{n-d-1}\right\}$, we conclude that $V_{\mathfrak{L}}$ contains all homogeneous polynomials of the form

$$
l_{i_{1}} l_{i_{2}} \ldots l_{i_{n-d-1}} u_{k}, \quad \text { for } 0 \leq k \leq d \text {. }
$$

From that we deduce that $V_{\mathfrak{L}}$ contains all homogeneous polynomials of the form $l_{i_{1}} l_{i_{2}} \ldots l_{i_{n-d-2}} u_{k} u_{j}$, where $j, k \in[n] \backslash\left\{i_{1}, \ldots, i_{n-d-1}\right\}$. Continuing along the same lines, we derive by induction that $V_{\mathfrak{L}}$ contains $H$ Poly $(n-d, d+1)$.

For an arbitrary spanning $S$, the cardinality of $\mathcal{B}_{N}$ is at most $\left(\begin{array}{c}N-1 \\ d\end{array}\right)=\operatorname{dim} \mathfrak{R a t}(S)$. Furthermore, if $S$ is not strongly non-degenerate the cardinality of $\mathcal{B}_{N}$ is strictly smaller than $\left(\begin{array}{c}N-1 \\ d\end{array}\right)$, as there will be linear dependencies among the standard measures on the simplices in $B_{N}$. Therefore, $\operatorname{dim} \mathfrak{F}^{\Delta}(S)<\operatorname{dim} \mathfrak{R a t}(S)$.

We define the square matrix $M a t_{\mathfrak{L}}$ of size $\left(\begin{array}{l}n \\ d\end{array}\right)$ with entries being coefficients of the above products of linear forms w.r.t. the standard monomial basis in $\mathrm{HPol}(n-d, d+$ 1).

Lemma 18 The determinant of Mat $\mathfrak{L}$ is proportional to the product of the determinants of all $(d+1)$-tuples $\left(l_{i_{1}}, l_{i_{2}}, \ldots, l_{i_{d+1}}\right), i_{1}<i_{2}<\ldots<i_{d+1}$. (By the determinant of a $(d+1)$-tuple of vectors in $\mathbb{R}^{d+1}$ with a fixed basis we mean the determinant of the matrix formed by the coordinates of these vectors in a chosen basis.)

Proof Indeed, $\operatorname{det}\left(M a t_{\mathfrak{L}}\right)$ is a form of degree $(d+1)\left(\begin{array}{l}n \\ d\end{array}\right)$ in the coefficients of the linear forms $l_{1}, \ldots, l_{n}$. Thus the product $\prod_{i_{1}, \ldots, i_{d+1}} \operatorname{det}\left(l_{i_{1}}, l_{i_{2}}, \ldots, l_{i_{d+1}}\right)$ has the same degree as $\operatorname{det}\left(M a t_{\mathfrak{L}}\right)$. Therefore it suffices to show that $\operatorname{det}\left(M_{a t_{\mathfrak{L}}}\right)$ vanishes as soon as some of $\operatorname{det}\left(l_{i_{1}}, l_{i_{2}}, \ldots, l_{i_{d+1}}\right)$ vanishes. (Observe that all polynomials $\operatorname{det}\left(M a t_{\mathfrak{L}}\right)$ are coprime.) Without loss of generality, assume that $l_{1}$ is a linear combination of $l_{2}, \ldots, l_{d+1}$. But 
then the column of Mat $\mathfrak{L}$ corresponding to the $(n-d)$-tuple $(1, d+2, d+3, \ldots, n)$ will be a linear combination of those corresponding to $(2, d+2, d+3, \ldots, n), \ldots(d+$ $1, d+2, d+3, \ldots, n)$.

Proof of Corollary 11 As we mentioned above, $\mathfrak{M}(S)$ is isomorphic to $\mathfrak{F}(S)$ and, analogously, $\mathfrak{M}^{\Delta}(S)$ is isomorphic to $\mathfrak{F}^{\Delta}(S)$. Thus, if we prove the equality $\mathfrak{F}(S)=$ $\mathfrak{F}^{\Delta}(S)$, then we get $\mathfrak{M}(S)=\mathfrak{M}^{\Delta}(S)$. By Lemma 17 , the space $\mathfrak{F}^{\Delta}(S)$ coincides with the linear space of all rational functions with the numerator an arbitrary polynomial of degree at most $N-d-1$ and the denominator $\Phi_{S}(\mathbf{u})$ equal to the product of all linear forms dual to all vertices in $S$. By Proposition 6 an arbitrary function in $\mathfrak{F}(S)$ is a rational function with denominator of desired form and numerator of degree at most $N-d-1$, for obvious reasons - take an arbitrary dissection and sum over its simplices. Since all such functions are already in $\mathfrak{F}^{\Delta}(S)$ we are done.

Proof of Theorem 13 Given a strongly non-degenerate set $S=\left\{\mathbf{v}_{1}, \ldots, \mathbf{v}_{N-1}, \mathbf{v}_{N}\right\}$ and the Fantappiè transform $R(\mathbf{u})=P(\mathbf{u}) / \Phi_{S}(\mathbf{u})$, where $\Phi_{S}(\mathbf{u})=\prod_{j=1}^{N} l_{j}(\mathbf{u})$, we want to solve the inverse moment problem. (It is easy to obtain $P(\mathbf{u})$ from information on the moments of order at most $N-d-1$ using Lemma 12.)

To solve the latter inverse problem using Corollary 3 , we need to find an appropriate set of weights $\mathbf{w}=\left\{w_{i_{1}, \ldots, i_{d}}\right\}, i_{1}<i_{1}<\ldots<i_{d}$, where $w_{i_{1}, \ldots, i_{d}}$ is the weight (recall Definition 1.14) of the $d$-dimensional simplex $\operatorname{conv}\left(\mathbf{v}_{i_{1}}, \mathbf{v}_{i_{2}}, \ldots, \mathbf{v}_{i_{d}}, \mathbf{v}_{N}\right)$ so that

$$
\sum_{i_{1}<i_{2}<\ldots<i_{d}} \frac{w_{i_{1}, \ldots, i_{d}}}{l_{i_{1}}(\mathbf{u}) l_{i_{2}}(\mathbf{u}) \ldots l_{i_{d}}(\mathbf{u}) l_{N}(\mathbf{u})}=\frac{P(\mathbf{u})}{l_{1}(\mathbf{u}) l_{2}(\mathbf{u}) \ldots l_{N}(\mathbf{u})}
$$

Clearing the denominators, we get the equation

$$
\sum_{i_{1}<i_{2}<\ldots<i_{d}} w_{i_{1}, \ldots, i_{d}} l_{j_{1}}(\mathbf{u}) l_{j_{2}}(\mathbf{u}) \ldots l_{j_{N-d-1}}(\mathbf{u}) l_{N}(\mathbf{u})=P(\mathbf{u})
$$

where $\left\{j_{1}, \ldots j_{N-d-1}\right\}=\{1,2, \ldots, N-1\} \backslash\left\{i_{1}, \ldots, i_{d}\right\}$. The latter equation is obviously equivalent to the system of linear equations

$$
\text { Mat }_{S} \cdot \mathbf{w}=\left(p_{I_{1}}, \ldots, p_{I_{t}}\right), \quad \text { where } P(\mathbf{u})=\sum_{I} p_{I} \mathbf{u}^{I},
$$

and $\mathbf{w}=\left\{w_{i_{1}, \ldots, i_{d}}\right\}$ is the vector consisting of the weights of all simplices containing $\mathbf{v}_{N}$.

Theorem 13 solves the inverse moment problem for strongly non-degenerate spanning set $S$. We can make this solution more explicit by giving a closed formula for the inverse matrix $\mathrm{Mat}_{S}^{-1}$. To do this, we introduce an extra variable $u_{0} \in \mathbb{R}$ and identify the space $\operatorname{Pol}(N-d-1, d)$ with the space $H \operatorname{Pol}(N-d-1, d+1)$ of homogeneous forms of degree $N-d-1$ in $d+1$ variables $\left(u_{0}, u_{1}, \ldots, u_{d}\right)$. We homogenize each linear form $l_{i}(\mathbf{u})$ in $\mathfrak{L}$ as $l_{i}\left(\mathbf{u}, u_{0}\right)=u_{0}-\left\langle\mathbf{v}_{i}, \mathbf{u}\right\rangle$. (The matrix Mat remains unchanged.) 
We also need the following $(d+1) \times(N-1)$ matrix $\mathbb{L}$

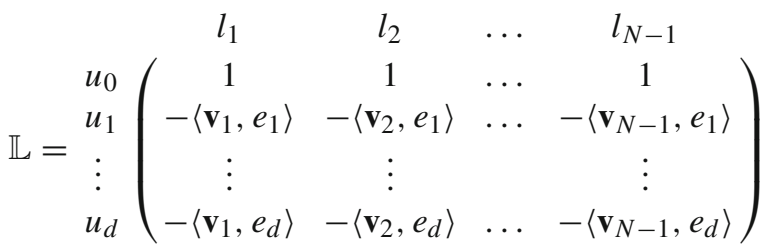

associated with $\mathrm{Mat}_{S}$. For all possible subsets $\mathbf{i}[d]=\left\{i_{1}, \ldots, i_{d}\right\}, i_{1}<i_{2}<\cdots<i_{d}$ of $d$ distinct columns of $\mathbb{L}$, consider the linear in $\mathbf{u}$ function $\mathbb{L}_{\mathbf{i}[d]}(\mathbf{u})$ given by:

$$
\mathbb{L}_{\mathbf{i}[d]}(\mathbf{u})=\operatorname{det}\left[\begin{array}{cccc}
u_{0} & 1 & \ldots & 1 \\
u_{1} & -\left\langle\mathbf{v}_{i_{1}}, e_{1}\right\rangle & \ldots & -\left\langle\mathbf{v}_{i_{d}}, e_{1}\right\rangle \\
\vdots & & \ldots & \\
u_{d} & -\left\langle\mathbf{v}_{i_{1}}, e_{d}\right\rangle & \ldots & -\left\langle\mathbf{v}_{i_{d}}, e_{d}\right\rangle
\end{array}\right]
$$

Denote by $\mathbb{L}_{\mathbf{i}[d], j}$ the coefficient of $u_{j}$ in the linear form $\mathbb{L}_{\mathbf{i}[d]}(\mathbf{u})$. For each $1 \leq j \leq$ $N-1$, define $\mathbb{L}(j, \mathbf{i}[d])$ as

$$
\mathbb{L}(j, \mathbf{i}[d])=\operatorname{det}\left[\begin{array}{cccc}
1 & 1 & \ldots & 1 \\
-\left\langle\mathbf{v}_{j}, e_{1}\right\rangle & -\left\langle\mathbf{v}_{i_{1}}, e_{1}\right\rangle & \ldots & -\left\langle\mathbf{v}_{i_{d}}, e_{1}\right\rangle \\
\vdots & & \ldots & \\
-\left\langle\mathbf{v}_{j}, e_{d}\right\rangle & -\left\langle\mathbf{v}_{i_{1}}, e_{d}\right\rangle & \ldots & -\left\langle\mathbf{v}_{i_{d}}, e_{d}\right\rangle
\end{array}\right]
$$

Note that if $j \notin \mathbf{i}[d]$ then $\mathbb{L}(j, \mathbf{i}[d]) \neq 0$, as by the assumption of strong nondegeneracy of $S$ the corresponding $d+1$ linear forms are linearly independent. On the other hand, if $j \in \mathbf{i}[d]$, then we have $\mathbb{L}(j, \mathbf{i}[d])=0$.

The matrix $\mathrm{Mat}_{S}^{-1}$ has the following explicit description.

Lemma 19 For each $(N-d-1)$-tuple of forms $\left\{l_{j_{1}}, l_{j_{2}}, \ldots, l_{j_{N-d-1}}\right\}$, set $\mathbf{i}[d]=$ $\left\{i_{1}, \ldots, i_{d}\right\}=[N-1] \backslash\left\{j_{1}, \ldots, j_{N-d-1}\right\}$, where $i_{1}<i_{2} \cdots<i_{d}$. Then,

$$
\left.\begin{array}{c}
\ldots a t_{S}^{-1}=l_{j_{1}} \ldots l_{j_{N-d-1}} \\
\vdots
\end{array} \quad \begin{array}{ccc} 
& u_{0}^{n_{0}} u_{1}^{n_{1}} \ldots u_{d}^{n_{d}} & \ldots \\
& \frac{\prod_{j=0}^{d} \mathbb{L}_{\mathbf{i}[d], j}^{n_{j}}}{\prod_{k=1}^{N-d-1} \mathbb{L}\left(j_{k}, \mathbf{i}[d]\right)} & \\
& \ldots &
\end{array}\right) .
$$

Proof of Lemma 19 In order to show that $\mathrm{Mat}_{S}^{-1}$ defined by (3.4) is indeed the inverse of $M a t_{S}$ we need to verify that $M a t_{S}^{-1} \cdot M_{a} t_{S}$ is the identity operator on $V_{\mathfrak{L}}$.

Let $\mathbf{e}^{\prime}$ be the standard basis vector of $V_{\mathfrak{L}}$ corresponding to the product of linear forms $l_{j_{1}^{\prime}} \ldots l_{j_{N-d-1}^{\prime}}$. Then $\mathrm{Mat}_{S} \cdot \mathbf{e}^{\prime}$ is the vector consisting of the monomial coefficients of the homogeneous form $l_{j_{1}^{\prime}} \ldots l_{j_{N-d-1}^{\prime}}$ in the variables $u_{0}, \ldots, u_{d}$. Let $\mathbf{e}^{T}$ be the 
row vector of $\mathrm{Mat}_{S}^{-1}$ corresponding to the product $l_{j_{1}} \ldots l_{j_{N-d-1}}$. We note that in $\mathbf{e}^{T} \cdot\left(\mathrm{Mat}_{S} \cdot \mathbf{e}^{\prime}\right)$ one can factor out the common denominator $\prod_{k=1}^{N-d-1} \mathbb{L}\left(j_{k}, \mathbf{i}[d]\right)$ of all fractions in $\mathbf{e}^{T}$; the remaining factor is of the form

$$
\sum_{\substack{I=\left(n_{0}, \ldots, n_{d}\right) \\|I|=N-d-1}} \operatorname{Mat}_{S}\left[\mathbf{u}^{I}, \mathbf{e}^{\prime}\right] \prod_{j=0}^{d} \mathbb{L}_{\mathbf{i}[d], j}^{n_{j}}=l_{j_{1}^{\prime}}\left(\mathbb{L}_{\mathbf{i}[d]}(\mathbf{u})\right) \ldots l_{j_{N-d-1}^{\prime}}\left(\mathbb{L}_{\mathbf{i}[d]}(\mathbf{u})\right) .
$$

Note that $l_{j_{k}^{\prime}}\left(\mathbb{L}_{\mathbf{i}[d]}(\mathbf{u})\right)=\mathbb{L}\left(j_{k}^{\prime}, \mathbf{i}[d]\right)$ for $1 \leq k \leq N-d-1$, i.e., this holds for all terms in the product on the right-hand side of (3.5). Hence, if $\mathbf{e} \neq \mathbf{e}^{\prime}$ then $\mathbf{e}^{T}$. $\left(\mathrm{Mat}_{S} \cdot \mathbf{e}^{\prime}\right)=0$, as among $j_{1}^{\prime}, \ldots, j_{N-d-1}^{\prime}$ one can find $j^{\prime} \in \mathbf{i}[d]$ with $\mathbb{L}\left(j^{\prime}, \mathbf{i}[d]\right)=0$. On the other hand, if $\mathbf{e}$ and $\mathbf{e}^{\prime}$ coincide, then the right-hand side of (3.5) is equal to $\prod_{k=1}^{N-d-1} \mathbb{L}\left(j_{k}, \mathbf{i}[d]\right)$. Dividing by the common denominator of the fractions in $\mathbf{e}$, we obtain $\mathbf{e}^{T} \cdot\left(\right.$ Mat $\left._{S} \cdot \mathbf{e}\right)=1$.

\section{Inverse moment problem for weakly non-degenerate $S$}

Given an arbitrary spanning set $S=\left\{\mathbf{v}_{1}, \mathbf{v}_{2}, \ldots, \mathbf{v}_{N}\right\}$, consider the linear space $\Theta(S) \subseteq \operatorname{Pol}(N-d-1, d)$ spanned by all products $l_{j_{1}} l_{j_{2}} \ldots l_{j_{N-d-1}}, j_{1}<j_{2}<$ $\ldots<j_{N-d-1}$. The next statement explains why we can extend our solution of the inverse moment problem from the case of strongly non-degenerate $S$ to the case of weakly non-degenerate $S$.

Lemma $20 \Theta(S)=\operatorname{Pol}(N-d-1, d)$ if and only if $S$ is weakly non-degenerate, i.e., each $(d+2)$-tuple of points of $S$ is spanning.

Proof We have $N$ (non-homogeneous) linear forms $l_{1} \ldots, l_{N}$ in variables $\mathbf{u}=$ $\left(u_{1}, \ldots, u_{d}\right)$ and the linear space $V_{\mathfrak{L}}$ spanned by all possible products of $(N-d-1)$ tuples of distinct forms. We need to investigate whether $V_{\mathfrak{L}}$ coincides with $\operatorname{Pol}(N-$ $d-1, d)$. Homogenizing, we consider the same question for the linear homogeneous forms and the homogeneous polynomials of degree $N-d-1$ in variables $\left(u_{0}, u_{1}, \ldots, u_{d}\right)$.

First assume that there are $d+2$ linear forms $l_{1}, \ldots, l_{d+2}$ which are not spanning. Then one can find a non-zero vector $\mathbf{z}_{0} \in \mathbb{R}^{d+1}$, such that $l_{1}(z)=\cdots=l_{d+2}(z)=0$. Note that each product of $N-d-1$ different forms chosen from $l_{1}, \ldots, l_{N}$ contains at least one form among $\left\{l_{1}, \ldots, l_{d+2}\right\}$. Therefore any linear combination of products of $N-d-1$ forms vanishes at $\mathbf{z}_{0}$. Thus $V_{\mathfrak{L}}$ cannot coincide with $H \operatorname{Pol}(N-d-1, d+1)$.

Conversely, assume that every $(d+2)$-tuple of distinct forms among $l_{1}, \ldots, l_{N}$ is spanning. First, we notice that $H \operatorname{Pol}(N-d-1, d+1)$ can be spanned by the all possible products of $N-d-1$ linear forms (not necessarily pairwise distinct). Indeed, since first $d+2$ forms span the dual space of $\mathbb{R}^{d+1}$, we can express each variable $x_{i}$ as a linear combination of these forms. Therefore every monomial of degree $N-d-1$ can be expressed as a linear combination of products of $N-d-1$ forms. 
Now we show that each product of $N-d-1$, not necessarily distinct, forms can be expressed as a linear combination of the products of distinct ones. Assume the contrary and consider monomials $l_{1}^{i_{1}} \ldots l_{N}^{i_{N}}$ of degree $N-d-1$ which cannot be expressed as a linear combination of products with all distinct forms. Among those monomials we take a monomial $\mathbf{m}=l_{1}^{k_{1}} \ldots l_{N}^{k_{N}}$ having the maximal number of distinct forms in the product. Since $\mathbf{m}$ is not a product of all distinct forms, it should contain a form $l_{i}$ in some power $k_{i} \geq 2$. Given that $k_{i} \geq 2$ and the degree of $\mathbf{m}$ is $N-d-1$, one can find $d+2$ distinct forms $l_{i_{1}}, \ldots, l_{i_{d+2}}$ that do not appear in $\mathbf{m}$. Since any $d+2$ of our forms span the dual space of $\mathbb{R}^{d+1}$, we can express $l_{i}$ as a linear combination of $l_{i_{1}}, \ldots, l_{i_{d+2}}$. Now rewrite $\mathbf{m}$ as $\left(\alpha_{1} \cdot l_{i_{1}}+\cdots+\alpha_{d+2} \cdot l_{i_{d+2}}\right) l_{1}^{k_{1}} \ldots l_{i}^{k_{i}-1} \ldots l_{N}^{k_{N}}$, where $\alpha_{1} \cdot l_{i_{1}}+\cdots+\alpha_{d+2} \cdot l_{i_{d+2}}=l_{i}$. Thus we get an expression of $\mathbf{m}$ as a linear combination of monomials $\alpha_{j} \cdot l_{i_{j}} l_{1}^{k_{1}} \ldots l_{i}^{k_{i}-1} \ldots l_{N}^{k_{N}}$, where each such monomial has more distinct forms than $\mathbf{m}$. Each of such monomials can be expressed as a linear combination of products of all distinct forms, since $\mathbf{m}$ was chosen as a monomial with the maximal possible number of distinct forms, which cannot be expressed in such a way. This is a contradiction. Therefore $\mathbf{m}$ can also be expressed as a linear combination of products of all distinct forms.

Below we consider the inverse problem for a weakly non-degenerate $S$, using notation from (3.1) and (3.3). Here we no longer have a natural basis of all simplices sharing a common vertex $\mathbf{v}_{N}$. Because of that we need to consider all $N$ points and include one more linear form $l_{N}$ into the corresponding matrix $\mathbb{L}$. Slightly abusing our notation, we denote by $\mathbb{L}$ the same matrix as before, although it contains one more (last) column corresponding to $\mathbf{v}_{N}$. Similarly to notation (3.3), for a given set $J$ of $d+1$ linear forms, we denote by $\mathbb{L}(J)$ the determinant of the corresponding $(d+1) \times(d+1)$-minor of $\mathbb{L}$.

We introduce the extended $\left(\begin{array}{c}N-1 \\ d\end{array}\right) \times\left(\begin{array}{c}N \\ d+1\end{array}\right)$-matrix $\widetilde{M a t}_{S}$ with columns consisting of the coefficients of the homogeneous polynomial $l_{i_{1}}(\mathbf{u}) \ldots l_{i_{N-d-1}}(\mathbf{u})$ with respect to the monomial basis in the variables $\left(u_{0}, u_{1}, \ldots, u_{d}\right)$. By Lemma 20, $\widetilde{\text { Mat }}_{S}$ has full rank, since it determines a surjective linear map onto $\mathrm{HPol}(N-d-1, d+1)$. Thus $\widetilde{M a t}_{S}$ has a maximal minor with a non-vanishing determinant. Formula (4.1) holds for the determinant of any maximal minor of $\widetilde{M a t}{ }_{S}$.

Lemma 21 Let $\mathfrak{S}$ be any set of $\left(\begin{array}{c}N-1 \\ d\end{array}\right)$ columns of $\widetilde{M a t}_{S}$. We label each column $T \in \mathfrak{S}$ by the corresponding subset of the linear forms $l_{1}, \ldots, l_{N}$ of cardinality $N-d-1$. Then the determinant of the maximal minor $\widetilde{M a t}_{S}(\mathfrak{S})$ formed by the columns of $\mathfrak{S}$ is given by:

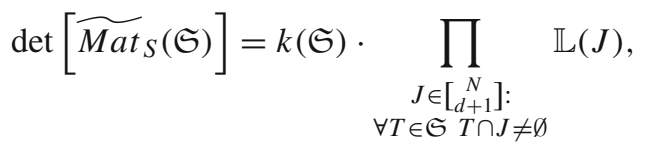

where $k(\mathfrak{S})$ is a constant (possibly equal to zero) depending only on the combinatorial structure of the $(N-d-1)$-tuples in the set $\mathfrak{S}$.

Proof Fix the set $\mathfrak{S}$ as above. In what follows, we treat both sides of (4.1) as complexvalued polynomials in $N \cdot(d+1)$ variables, these variables being the entries of matrix $\mathbb{L}$. 
We first show that every determinant $\mathbb{L}(J)$ divides $\operatorname{det}\left[\widetilde{M a t}_{S}(\mathfrak{S})\right]$. Indeed, let $J=\left\{j_{1}, \ldots, j_{d+1}\right\}$ be a set of $(d+1)$ forms which has a nonempty intersection with any $(N-d-1)$-tuple of forms in $\mathfrak{S}$. Let $\mathbf{z}=\left(z_{1}, \ldots, z_{N \cdot(d+1)}\right)$ be a zero of the polynomial $\mathbb{L}(J)$, which means that forms $l_{j_{1}}, \ldots, l_{j_{d+1}}$ comprised of the corresponding coordinates of $\mathbf{z}$ are linearly dependent. Therefore, there is a non-zero vector $\mathbf{u}_{0} \in \mathbb{R}^{d+1}$, such that $l_{j_{1}}\left(\mathbf{u}_{0}\right)=\cdots=l_{j_{d+1}}\left(\mathbf{u}_{0}\right)=0$. Consider the row vector $\left(\mathbf{u}_{0}^{I}\right)$ consisting of $\left(\begin{array}{c}N-1 \\ d\end{array}\right)$ homogeneous monomials of degree $N-d-1$ evaluated at $\mathbf{u}_{0}$. We notice that $\left(\mathbf{u}_{0}^{I}\right)$ is in the kernel of $\widetilde{M a t}_{S}(\mathfrak{S})$, as the product of $\left(\mathbf{u}_{0}^{I}\right)$ with each column vector $T \in \mathfrak{S}$ of $\widetilde{M a t}{ }_{S}(\mathfrak{S})$ is equal to $\prod_{j \in T} l_{j}\left(\mathbf{u}_{0}\right)$; and every set $T \in \mathfrak{S}$ contains at least one of the forms $l_{j_{1}}, \ldots, l_{j_{d+1}}$ in such a product. Thus $\operatorname{det}\left[\widetilde{\operatorname{Mat}}_{S}(\mathfrak{S})\right]$ also vanishes at such $\mathbf{z}$.

We recall a well-known fact (cf. e.g. [11, Theorem 61.1]) that

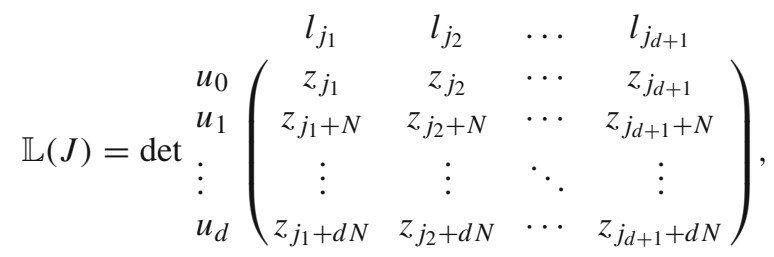

is an irreducible complex-valued polynomial in variables $z_{j_{1}}, z_{j_{2}} \ldots, z_{j_{d+1}+N d}$. Now if every zero of an irreducible polynomial $p\left(z_{1}, \ldots, z_{N(d+1)}\right)$ annihilates another polynomial $q\left(z_{1}, \ldots, z_{N(d+1)}\right)$, then $p$ divides $q$. We conclude that $\mathbb{L}(J)$ divides $\operatorname{det}\left[\widetilde{M a t}_{S}(\mathfrak{S})\right]$.

Using the fact that each $\mathbb{L}(J)$ is an irreducible polynomial and all $\mathbb{L}(J)$ 's are pairwise distinct (i.e., have distinct sets of projective zeros) we conclude that the product of $\mathbb{L}(J)$ 's in the right-hand side of (4.1) divides det $\left[\widetilde{M a t}_{S}(\mathfrak{S})\right]$.

Finally, the product of $\mathbb{L}(J)$ 's has the degree

$$
(d+1)\left|\left\{J \in\left[\begin{array}{c}
N \\
d+1
\end{array}\right] \mid \forall T \in \mathfrak{S} \quad T \cap J \neq \varnothing\right\}\right| .
$$

We observe that for each $T \in \mathfrak{S}$, the complementary set of $d+1$ forms cannot be taken as a feasible $J$. We notice further that these complements are the only exceptions for the choice of $J$. Therefore as a feasible $J$ we can pick any of $\left(\begin{array}{c}N \\ d+1\end{array}\right)(d+1)$-tuples except those $\left(\begin{array}{c}N-1 \\ d\end{array}\right)$ complements of a $T \in \mathfrak{S}$. Therefore, (4.2) equals

$$
(d+1)\left(\left(\begin{array}{c}
N \\
d+1
\end{array}\right)-\left(\begin{array}{c}
N-1 \\
d
\end{array}\right)\right)=(d+1)\left(\begin{array}{c}
N-1 \\
d+1
\end{array}\right)=(N-d-1)\left(\begin{array}{c}
N-1 \\
d
\end{array}\right)
$$

The latter expression coincides with the degree of the polynomial $\operatorname{det}\left[\widetilde{M a t}_{S}(\mathfrak{S})\right]$ (assuming that it is not a zero), as $\widetilde{M a t}_{S}(\mathfrak{S})$ has $\left(\begin{array}{c}N-1 \\ d\end{array}\right)$ columns and each entry is a homogeneous polynomial of degree $N-d-1$. 
Hence $\widetilde{M a t}_{S}(\mathfrak{S})$ coincides with the product of $\mathbb{L}(J)$ 's up to a constant factor which might vanish. This constant does not depend on the entries of matrix $\mathbb{L}$ and hence it is completely determined by the set $\mathfrak{S}$, regardless of the location of points of $S$ in $\mathbb{R}^{d}$.

Lemma 20 allows us to solve the inverse moment problem for a given weakly nondegenerate $S=\left\{\mathbf{v}_{1}, \ldots, \mathbf{v}_{N}\right\}$ in a certain linear space $\tilde{\mathfrak{M}}(S) \supseteq \mathfrak{M}^{\Delta}(S)$ of measures supported on $\operatorname{conv}(S)$. Namely, $\tilde{\mathfrak{M}}(S)$ is spanned by measures $\mu \in \tilde{\mathfrak{M}}(S)$ whose normalized moment generating functions $F_{\mu}(\mathbf{u})$ belong to $\mathfrak{R a t}(S)$, i.e. $F_{\mu}(\mathbf{u})=$ $P(\mathbf{u}) / \Phi_{S}(\mathbf{u})$, where $\Phi_{S}(\mathbf{u})=\prod_{j=1}^{N} l_{j}(\mathbf{u})$ and $P(\mathbf{u})$ is a polynomial of degree at most $N-d-1$. Indeed, by Lemma 20 any $R(\mathbf{u}) \in \mathfrak{R a t}(S)$ can be represented in the form

$$
R(\mathbf{u})=\sum_{i_{1}<i_{2}<\cdots<i_{d+1} \leq N} \frac{K_{i_{1} i_{2} \ldots i_{d+1}}}{l_{i_{1}} l_{i_{2}} \ldots l_{i_{d+1}}},
$$

with some real constants $K_{i_{1} i_{2} \ldots i_{d+1}}$. If $\mathbf{v}_{i_{1}}, \mathbf{v}_{i_{2}}, \ldots, \mathbf{v}_{i_{d+1}}$ span $\mathbb{R}^{d}$ then the term $\frac{K_{i_{1} i_{2} \ldots i_{d+1}}}{l_{i_{1}} l_{i_{2}} \ldots l_{d+1}}$ can be interpreted as the normalized moment generating function of an appropriately scaled standard measure of the $d$-dimensional simplex spanned by these vertices.

If $\mathbf{v}_{i_{1}}, \mathbf{v}_{i_{2}}, \ldots, \mathbf{v}_{i_{d+1}}$ only span a hyperplane $H$ in $\mathbb{R}^{d}$ then (4.3) corresponds to a singular (w.r.t. to the Lebesgue measure on $\mathbb{R}^{d}$ ) measure $\mu_{\delta}$ supported on $\delta=$ $\operatorname{conv}\left(\mathbf{v}_{i_{1}}, \ldots, \mathbf{v}_{i_{d+1}}\right)$. One way to define it as the weak limit of a sequence of (absolutely continuous with respect to the Lebesgue measure on $\mathbb{R}^{d}$ ) measures - the appropriately scaled standard measures $\mu_{\delta_{t}}$ of family of $d$-dimensional simplices $\delta_{t}$ which degenerate into $\delta$ when $t=0$. There is no loss in generality in assuming $K_{i_{1} i_{2} \ldots i_{d+1}}=1$, i.e., to deal with probability measures.

Proposition 22 Let $\mathbf{W}=\left\{\mathbf{w}_{1}, \ldots, \mathbf{w}_{d}, \mathbf{w}_{d+1}\right\}$ be a $(d+1)$-tuple of points in $\mathbb{R}^{d}$ such that $\mathbf{W}$ spans a hyperplane $H \subset \mathbb{R}^{d}$. Denote by $l_{\mathbf{w}_{1}}=1-\left\langle\mathbf{w}_{1}, \mathbf{u}\right\rangle, \ldots, l_{\mathbf{w}_{d+1}}=$ $1-\left\langle\mathbf{w}_{d+1}, \mathbf{u}\right\rangle$ the associated linear forms. There exists a unique measure $\mu_{\mathbf{W}}$ supported on $\delta=\operatorname{conv}(\mathbf{W})$ with the normalized moment generating function $F_{\mu_{\mathbf{W}}}(\mathbf{u})$ given by

$$
F_{\mu_{\mathbf{W}}}(\mathbf{u})=\frac{1}{l_{\mathbf{w}_{1}} l_{\mathbf{w}_{2}} \ldots l_{\mathbf{w}_{d+1}}} .
$$

Proof Without loss of generality assume that $\mathbf{W}=\left\{\mathbf{w}_{1}, \ldots, \mathbf{w}_{d}, \mathbf{w}_{d+1}\right\}$ is ordered in such a way that $\left\{\mathbf{w}_{1}, \ldots, \mathbf{w}_{d}\right\}$ span $H$. Then, $\delta_{t}$ is defined as $\delta_{t}=\operatorname{conv}\left(\delta, \mathbf{w}_{i_{d+1}}+\right.$ $t \mathbf{z}$ ), with $\mathbf{z}$ a unit normal to $H$, and $\mu_{\delta_{t}}$ as the uniform density probability measure supported on $\delta_{t}$. Then $\lim _{t \rightarrow 0} \mu_{\delta_{t}}=\mu_{\delta}$, where lim is understood in sense of weak convergence of distributions (measures), i.e., that $\lim _{t \rightarrow 0} \int f d \mu_{\delta_{t}}=\int f d \mu_{\delta}$ for any bounded, continuous real function on $\mathbb{R}^{d}$, cf. e.g. [10]. Then, this measure has compact support, and thus is determined by its moments, cf. e.g. [28, Proposition 3.2].

Remark 12 One can prove that the integration of a smooth compactly supported function $\phi$ with respect to the limiting measure $\mu_{\mathbf{W}}$ is given by the integration of $\phi$ over $\delta$ with a continuous piecewise linear weight function uniquely determined by $\delta$. Similar 
limits appear frequently in the theory of splines. Since we only need the existence of $\mu_{\mathbf{W}}$ we do not pursue this topic here.

Our solution of the inverse moment problem for the linear space $\tilde{\mathfrak{M}}(S)$ closely follows the pattern presented in Example 2. In other words, given a weakly nondegenerate $S$ and the set of moments up to order $N-d-1$ we

(i) produce the rational function $R(\mathbf{u}) \in \mathfrak{R a t}(S)$ with Taylor coefficients coinciding with the normalized moments;

(ii) represent $R(\mathbf{u})$ in the form (4.3);

(iii) for each term as in (4.4), determine the underlying measure supported on the (probably degenerate) convex hull of the vertices $\mathbf{v}_{i_{1}}, \mathbf{v}_{i_{2}}, \ldots, \mathbf{v}_{i_{d+1}}$.

We can now prove our central result claiming that $\mathfrak{M}^{\Delta}(S)=\mathfrak{M}(S)$ for a weakly non-degenerate $S$.

Proof of Theorem 9 Theorem 9 is already settled in Corollary 11 for the case of strongly non-degenerate $S$. It remains to consider the case of weakly non-degenerate $S$. The denominator of the moment generating function $F_{\mathcal{P}}(\mathbf{u})$ for an arbitrary generalized polytope $\mathcal{P}$ with the vertex set $S$ is of the form $\Pi_{i=1}^{N} l_{i}$ by Proposition 6 , and its numerator belongs to $\operatorname{Pol}(N-d-1, d)$. As $S$ is weakly non-degenerate, $F_{\mathcal{P}}(\mathbf{u})$ can be written as a linear combination of the fractions as in (4.3), where $\left(i_{1}, i_{2}, \ldots i_{d+1}\right)$ runs over the set of $(d+1)$-tuples of indices. If a $(d+1)$-tuple $l_{i_{1}}, l_{i_{2}}, \ldots l_{i_{d+1}}$ is spanning then $\frac{K}{l_{i_{1}} l_{i_{2}} \ldots l_{i} d+1}$ is the moment generating function of the measure supported on the simplex $\Delta$, determined by its denominator, with the uniform density $K / d ! \operatorname{Vol}(\Delta)$. By Proposition 22, if a $(d+1)$-tuple $l_{i_{1}}, l_{i_{2}}, \ldots l_{i_{d+1}}$ is not spanning then $\frac{K}{l_{i_{1}} l_{i_{2}} \ldots l_{i_{d+1}}}$ is the moment generating function of a singular measure supported on a degenerate simplex. As $\mathcal{P}$ is a generalized polytope, its standard measure has no singular components. Therefore, no degenerate simplices can appear in its decomposition.

Remark 13 The latter proof demonstrates that if one starts from the set of moments of the standard measure $\mu$ of a polytope with the vertex set $S$ then we never obtain degenerate simplices while solving the inverse moment problem. This is why $\mathfrak{M}^{\Delta}(S)=\mathfrak{M}(S)$. However, an explicit description of $\mathfrak{F}^{\Delta}(S)$ for a general weakly non-degenerate $S$ is missing at present. For concrete Examples 3 and 4, we give these descriptions below.

Our final result computes $\operatorname{dim} \mathfrak{M}^{\Delta}(S)$ and describes a procedure to construct a basis for $\mathfrak{M}^{\Delta}(S)$.

Proposition 23 Let $S=\left\{\mathbf{v}_{1}, \ldots, \mathbf{v}_{N}\right\} \subset \mathbb{R}^{d}$ be an arbitrary weakly non-degenerate spanning set. Then

(i) $\operatorname{dim} \mathfrak{M}^{\Delta}(S)=\left(\begin{array}{c}N-1 \\ d\end{array}\right)-\sharp_{\text {deg }}$ where $\sharp_{\text {deg }}$ is the number of degenerate simplices, i.e., the number of non-spanning $(d+1)$-tuples of points of $S$.

(ii) If $\delta$ is a degenerate d-dimensional simplex with vertices in $S \backslash\left\{\mathbf{v}_{i}\right\}$ then there is exactly one linear dependence among the standard measures of all d-dimensional simplices on $\mathbf{v}_{i}$ and $d$ vertices of $\delta$.

(iii) The standard measure of any d-dimensional simplex on $\mathbf{v}_{i}$ is contained in at most one dependence as in (ii). 
(iv) For any vertex $\mathbf{v}_{i}$, one can construct a (in general, non-unique) basis $\mathcal{B}_{i}$ of $\mathfrak{M}^{\Delta}(S)$ consisting of standard measures of d-dimensional simplices on $\mathbf{v}_{i}$, as follows.

(a) Start from the set $\mathcal{B}_{i}$ of the d-dimensional simplices on $\mathbf{v}_{i}$.

(b) For each degenerate simplex $\delta$ not containing $\mathbf{v}_{i}$, remove from $\mathcal{B}_{i}$ the standard measure of an arbitrary simplex on $\mathbf{v}_{i}$ from the corresponding to $\delta$ linear dependence, $c f$. (i).

Thus we obtain $\left(\begin{array}{c}N-1 \\ d\end{array}\right)-\sharp$ deg standard measures of d-dimensional simplices, forming a basis of $\mathfrak{M}^{\Delta}(S)$.

Proof To prove (i), notice that $\operatorname{dim} \tilde{\mathfrak{M}}(S)=\operatorname{dim} \mathfrak{R a t}(S)=\left(\begin{array}{c}N-1 \\ d\end{array}\right)$. As well, $\tilde{\mathfrak{M}}(S)=$ $\mathfrak{M}(S) \oplus \mathfrak{M}_{\text {deg }}(S)$, where $\mathfrak{M}_{\text {deg }}(S)$ is the linear span of the measures $\mu_{\delta}^{(1)}$ with $\delta$ running over the set of all degenerate simplices spanned by $(d+1)$-tuples of dependent vertices in $S$, cf. Proposition 22. Observe that these measures $\mu_{\delta}^{(1)}$ are linearly independent, as each degenerate simplex defines a singular measure supported in a proper hyperplane, and these hyperplanes differ for different degenerate simplices. We are done with (i).

Let $\Sigma_{0}$ be a dependent $d+1$-subset of $S$, and $\delta=\operatorname{conv}\left(\Sigma_{0}\right)$ be as in (ii). Then $\delta$ spans a hyperplane $H_{0}$. As each $d$-dimensional simplex on $\sigma_{0}:=\mathbf{v}_{i}$ and $d$ vertices from $\Sigma_{0}$ is uniquely defined by the latter, it suffices to analyze dependencies between the standard measures of $d-1$-simplices with vertices in $\Sigma_{0}$.

We can view $\Sigma_{0}$ as a weakly non-degenerate subset in $\mathbb{R}^{d-1} \cong H_{0}$. By (i), we have $\operatorname{dim} \mathfrak{M}^{\Delta}\left(\Sigma_{0}\right)=\left(\begin{array}{c}d \\ d-1\end{array}\right)-\sharp_{d e g}\left(\Sigma_{0}\right)$. If $\Sigma_{0}$ is strongly non-degenerate as a subset of $H_{0} \cong \mathbb{R}^{d-1}$, i.e. $\sharp$ deg $\left(\Sigma_{0}\right)=0$, then $\operatorname{dim} \mathfrak{M}^{\Delta}\left(\Sigma_{0}\right)=d$, i.e., there is exactly one linear dependence between the standard measures of $d-1$-simplices with vertices in $\Sigma_{0}$, and we are done. Otherwise, $\Sigma_{0}=\left\{\sigma_{1}\right\} \cup \Sigma_{1}$, with $\Sigma_{1}$ spanning a hyperplane $H_{1}$ in $H_{0}$. Moreover, this can only happen if $d \geq 3$. Now, we can repeat the whole argument with $\sigma_{1}$ in place of $\sigma_{0}, \Sigma_{1}$ in place of $\Sigma_{0}$, and $H_{1}$ in place of $H_{0}$. Again, we either have $\Sigma_{1}$ strongly degenerate, and we are done, or we repeat this argument, etc., until we hit a strongly non-degenerate $\Sigma_{k}$, which is bound to happen, as the dimension goes down each iteration. This completes the proof of (ii).

Then, (iii) stems from the fact that the vertices of $d$-dimensional simplex on $\mathbf{v}_{i}$ distinct from $\mathbf{v}_{i}$ span a hyperplane, and the only possibility for a degenerate simplex $\delta$ as in (ii) is to lie in this hyperplane.

Finally, to prove (iv), observe that the set $\mathcal{B}_{i}^{\prime}$ of the standard measures of $d$-dimensional simplices containing a given vertex $\mathbf{v}_{i}$ always spans $\mathfrak{M}^{\Delta}(S)$, see Lemma 16. Now for each degenerate $d$-simplex $\delta$, we prune $\mathcal{B}_{i}^{\prime}$ by removing the standard measure of a simplex in the linear dependence corresponding to $\delta$. In view of (ii) and (iii) this process is well-defined and unambiguous. In the end we obtain $\left(\begin{array}{c}N-1 \\ d\end{array}\right)-\sharp$ deg standard measures of $d$-dimensional simplices. In view of (i) they form a basis of $\mathfrak{M}^{\Delta}(S)$, as claimed.

Remark 14 The above discussions show that the columns of $\widetilde{M a t}_{S}$ corresponding to degenerate simplices must necessarily be included in any non-vanishing maximal minor $\widetilde{\operatorname{Mat}}_{S}(\mathfrak{S})$.

We conclude our discussion of the weakly non-degenerate case with two examples. 

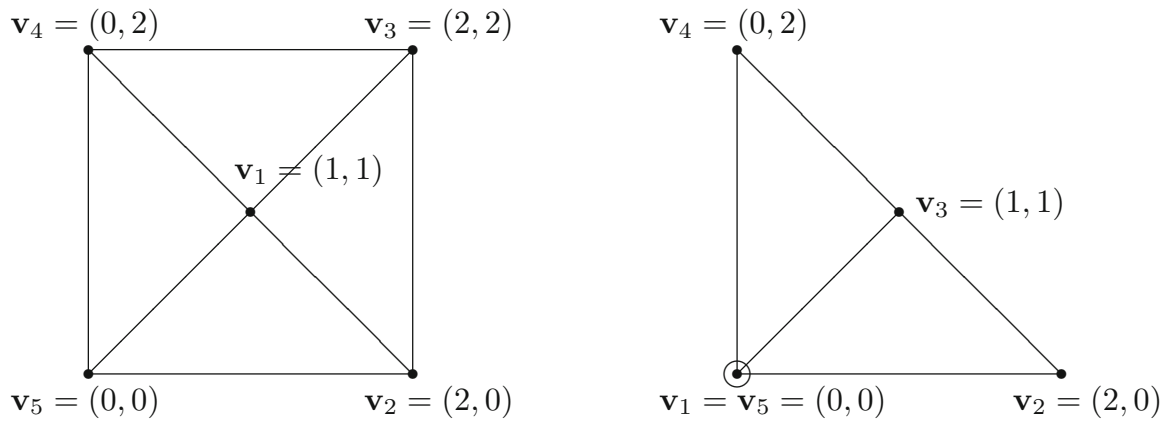

Fig. 3 Vertices for Examples 3 and 4

Example 3 Let $S=\left\{\mathbf{v}_{1}, \mathbf{v}_{2}, \mathbf{v}_{3}, \mathbf{v}_{4}, \mathbf{v}_{5}\right\}$ where $\mathbf{v}_{1}=(1,1), \mathbf{v}_{2}=(2,0), \mathbf{v}_{3}=$ $(2,2), \mathbf{v}_{4}=(0,2), \mathbf{v}_{5}=(0,0)$ as on the left-hand side of Fig. 3. Then $l_{1}=$ $1-u_{1}-u_{2}, l_{2}=1-2 u_{1}, l_{3}=1-2 u_{1}-2 u_{2}, l_{4}=1-2 u_{2}, l_{5}=1$. Calculating the products $l_{i} l_{j}, i<j$ and taking their coefficients in the standard monomial basis of $\operatorname{Pol}(2,2)$ we obtain the following $6 \times 10$-matrix $\widetilde{M a t} S$

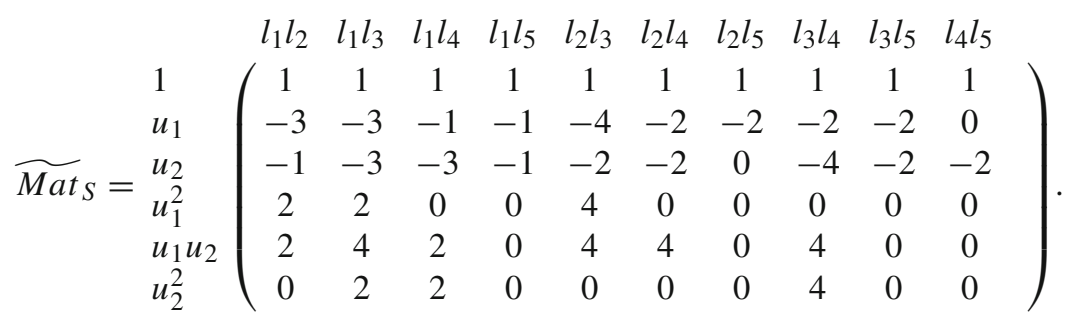

Its rank equals 6 and one of non-vanishing maximal minors consists of the columns with numbers $\mathfrak{S}=\{5,6,7,8,9,10\}$. (Recall that any non-vanishing maximal minor must include columns 6 and 9 corresponding to degenerate triples $\left(\mathbf{v}_{1}, \mathbf{v}_{3}, \mathbf{v}_{5}\right)$ and $\left(\mathbf{v}_{1}, \mathbf{v}_{2}, \mathbf{v}_{4}\right)$ resp.) The corresponding submatrix $\widetilde{M a t}{ }_{S}(\mathfrak{S})$ equals

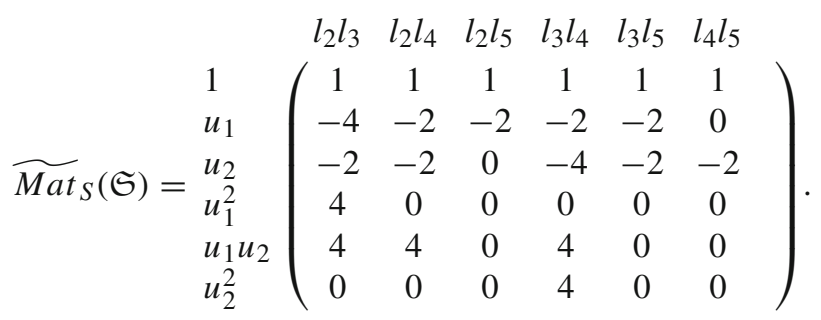


Further,

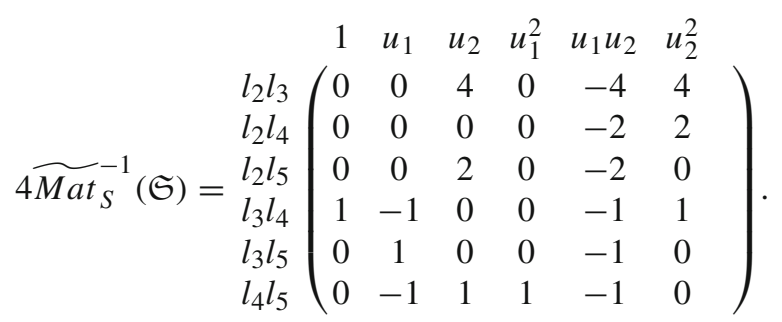

Thus, given an arbitrary rational function $R\left(u_{1}, u_{2}\right)=P\left(u_{1}, u_{2}\right) / \Phi_{S}\left(u_{1}, u_{2}\right)$, where $P\left(u_{1}, u_{2}\right)=a_{00}+a_{1,0} u_{1}+a_{0,1} u_{2}+a_{2,0} u_{1}^{2}+a_{11} u_{1} u_{2}+a_{02} u_{2}^{2}$ is any polynomial of degree at most 2 and $\Phi_{S}\left(u_{1}, u_{2}\right)=l_{1} l_{2} l_{3} l_{4} l_{5}$, we obtain

$$
\left\{\begin{array}{l}
w_{145}=-a_{10}-a_{20}-a_{11} \\
w_{135}=-\frac{1}{2}\left(a_{11}-a_{02}\right) \\
w_{134}=\frac{1}{2}\left(a_{01}-a_{11}\right) \\
w_{125}=\frac{1}{4}\left(a_{00}-a_{10}-a_{11}+a_{02}\right) \\
w_{124}=\frac{1}{4}\left(a_{10}-a_{11}\right) \\
w_{123}=-\frac{1}{4}\left(a_{10}-a_{01}-a_{20}+a_{11}\right)
\end{array}\right.
$$

Triangles $\Delta_{135}$ and $\Delta_{124}$ are degenerate which implies that if the original measure we are recovering is polygonal then $w_{135}=w_{124}=0$. Therefore the linear space of numerators $P\left(u_{1}, u_{2}\right)$ for the space $\mathfrak{F}^{\Delta}(S)$ in this example is given by the relation

$$
a_{01}=a_{11}=a_{02} .
$$

Our last example is more degenerate than the previous one, although still weakly non-degenerate. In fact, in this example $S$ is a multiset since $\mathbf{v}_{1}=\mathbf{v}_{5}$. It shows that our technique can be generalized to a certain class of multisets as well.

Example 4 Set $S=\left\{\mathbf{v}_{1}, \mathbf{v}_{2}, \mathbf{v}_{3}, \mathbf{v}_{4}, \mathbf{v}_{5}\right\}$, where $\mathbf{v}_{1}=\mathbf{v}_{5}=(0,0), \mathbf{v}_{2}=(2,0), \mathbf{v}_{3}=$ $(1,1), \mathbf{v}_{4}=(0,2)$ as on the right-hand side of Fig. 3. Then $l_{1}=l_{5}=1, l_{2}=$ $1-2 u_{1}, l_{3}=1-u_{1}-u_{2}, l_{4}=1-2 u_{2}$. Calculating all products $l_{i} l_{j}, i<j$ and taking their coefficients in the standard monomial basis of $\operatorname{Pol}(2,2)$, we obtain the following $6 \times 10$-matrix $\widetilde{M a t}_{S}$ :

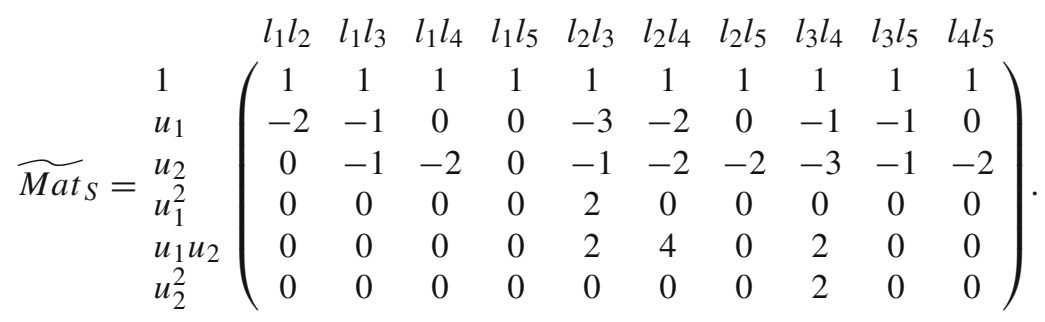


Its rank equals 6 and a non-vanishing maximal minor consists of the columns with numbers $\mathfrak{S}=\{1,3,4,5,6,8\}$. The corresponding submatrix $\widetilde{M a t}_{S}(\mathfrak{S})$ equals

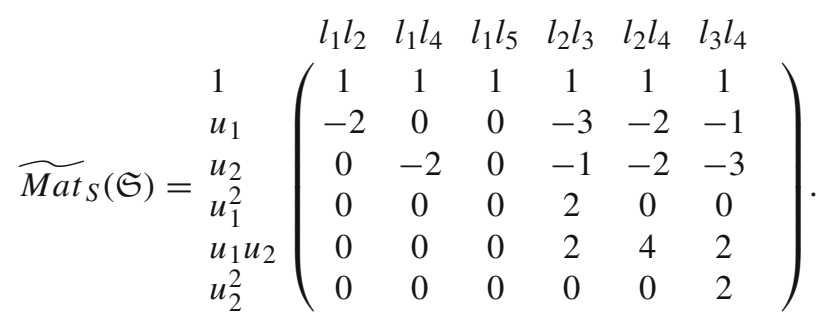

Further,

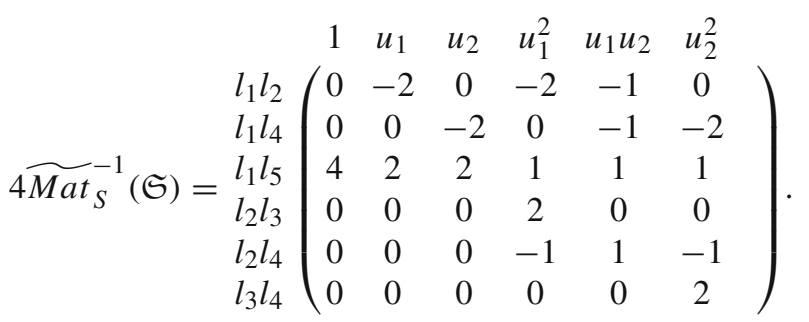

Thus, given an arbitrary rational function $R\left(u_{1}, u_{2}\right)=P\left(u_{1}, u_{2}\right) / \Phi_{S}\left(u_{1}, u_{2}\right)$, where $P\left(u_{1}, u_{2}\right)=a_{00}+a_{1,0} u_{1}+a_{0,1} u_{2}+a_{2,0} u_{1}^{2}+a_{11} u_{1} u_{2}+a_{02} u_{2}^{2}$ is any polynomial of degree at most 2 and $\Phi_{S}\left(u_{1}, u_{2}\right)=l_{1} l_{2} l_{3} l_{4} l_{5}$, we obtain

$$
\left\{\begin{array}{l}
w_{345}=-\frac{1}{4}\left(2 a_{10}+2 a_{20}+a_{11}\right) \\
w_{235}=-\frac{1}{4}\left(\left(2 a_{01}+a_{11}+2 a_{02}\right)\right. \\
w_{234}=4 a_{00}+2 a_{01}+2 a_{10}+a_{20}+a_{11}+a_{02} \\
w_{145}=2 a_{20} \\
w_{135}=-a_{10}+a_{11}-a_{20} \\
w_{125}=2 a_{02}
\end{array}\right.
$$

Notice that triangles $\Delta_{125}, \Delta_{135}, \Delta_{145}, \Delta_{234}$ are degenerate. If we know that the original measure we are recovering is polygonal then one should get $w_{125}=w_{135}=$ $w_{145}=w_{234}=0$. Therefore, the linear space of numerators $P\left(u_{1}, u_{2}\right)$ for the space $\mathfrak{F}^{\Delta}(S)$ in this example is given by the system of equations:

$$
\left\{\begin{array}{l}
a_{20}=0 \\
a_{10}=a_{11} \\
a_{02}=0 \\
4 a_{00}+2 a_{01}+3 a_{10}=0
\end{array}\right.
$$




\section{Remarks and open problems}

Remark 15 A weaker form of Corollary 4 (i.e., the rationality of $F_{\mathcal{P}}^{\rho}(\mathbf{u})$, but without the claim on the particular shape of the denominator) can be derived directly from (1.3) by using Stokes formula, along the lines of [7, Lemma 1].

Problem 2 Find an appropriate version of Theorem 2, applicable to non-simple and/or non-convex polytopes.

Remark 16 Choose an arbitrary basis $\left\{\Delta_{j}\right\}$ of $\mathfrak{M}^{\Delta}(S)$ consisting of the standard measures of simplices. The set $\left\{\Delta_{j}\right\}$ spans an integer lattice in $\mathfrak{M}^{\Delta}(S)$. (One can easily see that this lattice is invariantly defined independently of the choice of a basis of standard measures of simplices.) Denote by $\mathfrak{M}_{\mathbb{Z}}^{\Delta}(S)$ the space $\mathfrak{M}^{\Delta}(S)$ with the latter lattice. We can prove the following.

Proposition 24 Any generalized polytope $\mathcal{P} \in \mathcal{P}(S)$ with standard measure $\mu_{\mathcal{P}}$ corresponds to a rational point in $\mathfrak{M}_{\mathbb{Z}}^{\Delta}(S)$.

Proof As Conjecture 7 follows from [1, Theorem 1], one has $\mu_{\mathcal{P}} \in \mathfrak{M}^{\Delta}(S)$. One can easily show that $\mathcal{P}$ can be represented as the union of the closures of connected components of $\mathbb{R}^{d} \backslash H(S)$, where $H(S)$ is the hyperplane arrangement consisting of all hyperplanes spanned by $d$-tuples of points in $S$. (The converse is obviously not true.) Let $\tilde{S} \supseteq S$ be the extended set of vertices obtained by adding to $S$ all vertices of the hyperplane arrangement $H(S)$. Since each connected component in $\mathbb{R}^{d} \backslash H(S)$ is convex, it can be triangulated on $\tilde{S}$. Consider the space $\mathfrak{M}_{\mathbb{Z}}^{\Delta}(\tilde{S})$. Obviously, $\mu_{\mathcal{P}}$ is an integer point in $\mathfrak{M}_{\mathbb{Z}}^{\Delta}(\tilde{S})$. Also, $\mathfrak{M}_{\mathbb{Z}}^{\Delta}(S)$ is contained in $\mathfrak{M}_{\mathbb{Z}}^{\Delta}(\tilde{S})$ as a sublattice. Thus, if $\mu_{\mathcal{P}}$ belongs to $\mathfrak{M}_{\mathbb{Z}}^{\Delta}(S)$ it is a rational point there.

Problem 3 One can also define a rational convex cone $\mathfrak{P o s}(S) \subset \mathfrak{M}_{\mathbb{Z}}(S)$ by taking non-negative linear combinations of all $\mu_{\mathcal{P}}$, where $\mathcal{P}$ runs over the set of all generalized polytopes in $\mathcal{P}(S)$.

Conjecture 25 The rational cone $\mathfrak{P o s}(S)$ is uniquely determined by the oriented matroid associated to $S$.

We conclude this section with the following question.

Problem 4 Is it possible to describe the extremal rays of $\mathfrak{P o s}(S)$ ?

One can easily show that a simplex from $\mathcal{P}(S)$ spans an extremal ray of $\mathfrak{P o s}(S)$ if and only if it does not contain any points of $S$ distinct from its vertices. Problem 4 is apparently closely related to the problem of classification of combinatorial types of point arrangements, see e.g. [17] and references therein.

\subsection{Hyperplane arrangements and Laplace transform}

After the first version [19] of this text was released, it was pointed out to us that Laplace transform technique developed for studying hyperplane arrangements in [13] 
(see also [15, Sect. II.8]) and the corresponding knowledge accummulated simplify and strengthen a number of our results. Here we sketch the key ideas, leaving full details for another publication.

Let $\mathcal{P}$ be a generalized polytope. It is natural to homogenize its normalized moment generating function-the rational function $F_{\mathcal{P}}^{\rho}(\mathbf{u})$ from Proposition 6-so that its numerator and denominator become homogeneous, using an extra variable $\mathbf{u}_{0}$. E.g. this allows to account for the origin appearing as a vertex of $\mathcal{P}$. It also has a natural interpretation in terms of measures. Namely, embed $\mathcal{P}$ in the hyperplane $\left\{\mathbf{u} \mid \mathbf{u}_{0}=1\right\}$, and consider the cone spanned by $\mathcal{P}$; equip this cone with exponentially decaying in the direction $\mathbf{u}_{0}$ measure. Then the Laplace transform of this measure is the homogenization $\tilde{F}_{\mathcal{P}}(\tilde{\mathbf{u}})$ of $F_{\mathcal{P}}^{\rho}(\mathbf{u})$, where we denoted $\tilde{\mathbf{u}}:=\left(\mathbf{u}_{0}, \mathbf{u}_{1}, \ldots, \mathbf{u}_{d}\right)$.

Note that the denominator of $\tilde{F}_{\mathcal{P}}(\tilde{\mathbf{u}})$ is the product of powers of linear forms $\ell_{\mathbf{v}}(\tilde{\mathbf{u}})$, with $\mathbf{v} \in \mathcal{V}(\mathcal{P})$. The paper [13] associates to the hyperplane arrangement specified by the corresponding hyperplanes the algebra of rational functions generated by the reciprocials of the $\ell_{\mathbf{v}}$, endowed with the natural action of the polynomial differential operators. Then it proceeds to show that $\tilde{F}_{\mathcal{P}}(\tilde{\mathbf{u}})$ admits a decomposition into a sum of $\tilde{F}_{\Delta}(\tilde{\mathbf{u}})$, with $\Delta$ ranging through $d$-simplices with vertices in $\mathcal{V}(\mathcal{P})$, whenever $\tilde{F}(\tilde{\mathbf{u}})$ corresponds to a non-singular polynomial density measure. This in particular implies Conjecture 7, and much more.

Acknowledgements The second author is grateful to the Mathematics Department of Stockholm University for the hospitality in June 2011 when this project was initiated. The third author wants to acknowledge the hospitality of the School of Physical and Mathematical Sciences, Nanyang Technological University in April 2012 when this project was completed. We want to thank Sinai Robins and Michèle Vergne for numerous discussions of the topic. We acknowledge extremely helpful answers and comments on our questions on mathoverflow.net, in particular ones by David Eppstein, Dirk Lorenz, Igor Pak, David Speyer, and Gjergji Zaimi. Finally, the third author wants to thank late Mikael Passare (who unfortunately left us so early) for discussions of the properties of Fantappiè transformation and for pointing out reference [5] in September 2011.

Open Access This article is distributed under the terms of the Creative Commons Attribution 4.0 International License (http://creativecommons.org/licenses/by/4.0/), which permits unrestricted use, distribution, and reproduction in any medium, provided you give appropriate credit to the original author(s) and the source, provide a link to the Creative Commons license, and indicate if changes were made.

\section{References}

1. Akopyan, A., Bárány, I., Robins, S.: Algebraic vertices of non-convex polyhedra. Adv. Math. 308, 627-644 (2017)

2. Alekseevskaya, T.V., Gel'fand, I.M., Zelevinskiŭ, A.V.: Distribution of real hyperplanes and the partition function connected with it. Dokl. Akad. Nauk SSSR 297(6), 1289-1293 (1987)

3. Alekseyevskaya, T.: Bases in systems of simplices and chambers. ArXiv e-prints (1997). arXiv:math/9707218

4. Alekseyevskaya, T.V.: Combinatorial bases in systems of simplices and chambers. In: Proceedings of the 6th Conference on Formal Power Series and Algebraic Combinatorics (New Brunswick, NJ, 1994), pp. 15- 37 (1996)

5. Andersson, M., Passare, M., Sigurdsson, R.: Complex convexity and analytic functionals. Progress in Mathematics, vol. 225. Birkhäuser, Basel (2004)

6. Baldoni, V., Berline, N., De Loera, J.A., Köppe, M., Vergne, M.: How to integrate a polynomial over a simplex. Math. Comput. 80(273), 297-325 (2011) 
7. Barvinok, A.I.: Exponential integrals and sums over convex polyhedra. Funktsional. Anal. i Prilozhen. 26(2), 64-66 (1992)

8. Barvinok, A.: Integer points in polyhedra, Zurich Lectures in Advanced Mathematics. European Mathematical Society, EMS), Zürich (2008)

9. Beck, M., Robins, S.: Computing the Continuous Discretely: Integer-Point Enumeration in Polyhedra. Undergraduate Texts in Mathematics. Springer, New York (2007)

10. Billingsley, P.: Convergence of probability measures, Second. Probability and Statistics. Wiley., New York. A Wiley-Interscience Publication, Wiley Series in Probability and Statistics (1999)

11. Bôcher, M.: Introduction to Higher Algebra. Dover Publications Inc., New York (1964)

12. Brion, M.: Points entiers dans les polyèdres convexes. Ann. Sci. École Norm. Sup. (4) 21(4), 653-663 (1988)

13. Brion, M., Vergne, M.: Arrangement of hyperplanes. I. Rational functions and Jeffrey-Kirwan residue. Ann. Sci. École Norm. Sup. (4) 32(5), 715-741 (1999)

14. Brodsky, M.A.: On the uniqueness of the inverse potential problem for homogeneous polyhedrons. SIAM J. Appl. Math. 46(2), 345-350 (1986)

15. De Concini, C., Procesi, C.: Topics in Hyperplane Arrangements. Polytopes and Box-Splines. Universitext, Springer, New York (2011)

16. De Loera, J.A., Rambau, J., Santos, F.: Triangulations, Structures for Algorithms and Applications. Algorithms and Computation in Mathematics, vol. 25. Springer, Berlin (2010)

17. Fukuda, K., Miyata, H., Moriyama, S.: Complete enumeration of small realizable oriented matroids. ArXiv e-prints (2012). arXiv:1204.0645

18. Golub, G.H., Milanfar, P., Varah, J.: A stable numerical method for inverting shape from moments. SIAM J. Sci. Comput. 21(4), 1222-1243 (1999). (electronic)

19. Gravin, N., Pasechnik, D.V., Shapiro, B., Shapiro, M.: On moments of a polytope. ArXiv e-prints (2012). arXiv:1210.3193v1

20. Gravin, N., Lasserre, J.B., Pasechnik, D.V., Robins, S.: The inverse moment problem for convex polytopes. Discrete Comput. Geom. 48(3), 596-621 (2011). ArXiv eprint arXiv:1106.5723

21. Gustafsson, B., He, C., Milanfar, P., Putinar, M.: Reconstructing planar domains from their moments. Inverse Probl. 16(4), 1053-1070 (2000)

22. Lawrence, J.: Polytope volume computation. Math. Comput. 57(195), 259-271 (1991)

23. McCarthy, J.E., Putinar, M.: Positivity aspects of the Fantappiè transform. J. Anal. Math. 97, 57-82 (2005)

24. Milanfar, P., Putinar, M., Varah, J., Gustafsson, B., Golub, G.H.: Shape reconstruction from moments: theory, algorithms, and applications. In: Society of Photo-Optical Instrumentation Engineers (SPIE) Conference Series, pp. 406-416 (2000)

25. Pak, I.: Lectures on discrete and polyhedral geometry, UCLA (2008). http://www.math.ucla.edu/ pak/ book.htm

26. Pasechnik, D., Shapiro, B.: On polygonal measures with vanishing harmonic moments. J. d'Analyse Math. 123, 281-301 (2014). ArXiv eprint arXiv:1209.4014

27. Pukhlikov, A.V., Khovanskiǔ, A.G.: The Riemann-Roch theorem for integrals and sums of quasipolynomials on virtual polytopes. Algebra i Analiz 4(4), 188-216 (1992)

28. Putinar, M., Scheiderer, C.: Multivariate moment problems: geometry and indeterminateness. Ann. Sc. Norm. Super. Pisa Cl. Sci. (5) 5(2), 137-157 (2006)

29. Rudin, W.: Real and Complex Analysis, 3rd edn. McGraw-Hill Book Co., New York (1987)

30. Schönhardt, E.: Über die Zerlegung von Dreieckspolyedern in Tetraeder. Math. Ann. 98(1), 309-312 (1928)

31. Strakhov, V.N., Brodsky, M.A.: On the uniqueness of the inverse logarithmic potential problem. SIAM. J. Appl. Math. 46(2), 324-344 (1986) 